\title{
Spaceborne Polarimetric SAR Interferometry: Performance Analysis and Mission Concepts
}

\author{
Gerhard Krieger \\ Microwaves and Radar Institute, German Aerospace Centre (DLR) e.V., P.O. Box 1116, 82230 Wessling, Germany \\ Email: gerhard.krieger@dlr.de
}

Konstantinos Panagiotis Papathanassiou

Microwaves and Radar Institute, German Aerospace Centre (DLR) e.V., P.O. Box 1116, 82230 Wessling, Germany Email: kostas.papathanassiou@dlr.de

\author{
Shane R. Cloude \\ School of Electrical and Electronic Engineering, The University of Adelaide, Adelaide, SA 5005, Australia \\ Email: scloude@eleceng.adelaide.edu.au
}

Received 30 July 2004; Revised 3 January 2005

\begin{abstract}
We investigate multichannel imaging radar systems employing coherent combinations of polarimetry and interferometry (PolInSAR). Such systems are well suited for the extraction of bio- and geophysical parameters by evaluating the combined scattering from surfaces and volumes. This combination leads to several important differences between the design of Pol-InSAR sensors and conventional single polarisation SAR interferometers. We first highlight these differences and then investigate the Pol-InSAR performance of two proposed spaceborne SAR systems (ALOS/PalSAR and TerraSAR-L) operating in repeat-pass mode. For this, we introduce the novel concept of a phase tube which enables (1) a quantitative assessment of the Pol-InSAR performance, (2) a comparison between different sensor configurations, and (3) an optimization of the instrument settings for different Pol-InSAR applications. The phase tube may hence serve as an interface between system engineers and application-oriented scientists. The performance analysis reveals major limitations for even moderate levels of temporal decorrelation. Such deteriorations may be avoided in single-pass sensor configurations and we demonstrate the potential benefits from the use of future bi- and multistatic SAR interferometers.
\end{abstract}

Keywords and phrases: synthetic aperture radar, polarimetric SAR interferometry, bistatic radar, remote sensing, temporal decorrelation, forest parameter inversion.

\section{INTRODUCTION}

One of the key challenges facing synthetic aperture radar (SAR) remote sensing is to force evolution from highresolution qualitative imaging to accurate high-resolution quantitative measurement. However, quantitative estimation of relevant physical parameters from SAR data is in general nontrivial due to the fact that the radar measurables are not directly related to the desired parameters. Thus, the extraction of bio- and geophysical parameters often requires the inversion of scattering models that relate the radar observables to physical parameters of the scattering process. Due to the complexity of electromagnetic (EM) scattering

This is an open access article distributed under the Creative Commons Attribution License, which permits unrestricted use, distribution, and reproduction in any medium, provided the original work is properly cited. processes, even simple scattering models contain more parameters than the number of observables offered by a conventional single-frequency, single-polarisation SAR acquisition. One approach to reduce the number of unknowns is to utilise a priori information about the occurring scattering process and/or to introduce simplifying assumptions. The price to be paid is the restricted applicability in terms of validity range or transferability of the resulting inversion algorithms. A more promising approach is to extend the dimension of the observation vector by means of multiparameter SAR data acquisitions.

One very promising way to extend the observation space is the combination of interferometric and polarimetric observations. SAR interferometry is today an established technique for estimation of the height location of scatterers through the phase difference in images acquired from spatially separated apertures at either end of a baseline $[1,2,3]$. 
The sensitivity of the interferometric phase and coherence to spatial variability of vegetation height and density make the estimation of vegetation parameters from interferometric measurements at lower frequencies (C-, L-, or P-band) a challenge $[4,5,6,7]$. On the other hand, scattering polarimetry is sensitive to the shape, orientation, and dielectric properties of scatterers. This allows the identification and separation of scattering mechanisms of natural media by employing differences in the polarisation signature for purposes of classification and parameter estimation $[8,9]$. In polarimetric SAR interferometry (Pol-InSAR), both techniques are coherently combined to provide sensitivity to the vertical distribution of different scattering mechanisms [10, 11, 12]. Hence, it becomes possible to investigate the $3 \mathrm{D}$ structure of volume scatterers such as vegetation and ice, promising a breakthrough in radar remote sensing problems.

Regarding the range of natural volume scatterers, forest scatterers are the ones that obtained most of the scientific attention over the last years leading to impressive results. Indeed, accurate estimation of forest height from modelbased inversion of Pol-InSAR data has been demonstrated and validated over a large range of temperate and boreal regional test sites using airborne sensors $[12,13,14,15]$. The fact that forest height is the most important single forest parameter for ecological as well as for commercial applications $[16,17,18,19]$ and that it allows an unbiased forest biomass estimation [20,21] makes its estimation in terms of Pol-InSAR a key SAR technique. However, in order to evolve from local/regional to large-scale/global demonstrations and products-not only in forest applications-the implementation of Pol-InSAR technology in a spaceborne scenario is essential.

For Pol-InSAR applications, the performance criteria that apply to space-borne missions/sensors are different from the ones used in conventional and differential InSAR topographic mapping applications. For conventional InSAR DEM generation, the system performance is measured against the final height error-referred to a surface- that is composed of (a) the standard deviation dictated by the overall system coherence for a given imaging geometry and scatterer structure and (b) the height error introduced by the imaging geometry estimation. Pol-InSAR applications now deal with parameter estimation of natural volume scatterers based on the polarimetric diversity of InSAR observations (i.e., coherence and phase). Accordingly, one key criterion for the performance of a Pol-InSAR configuration is how strong the InSAR coherence and phase vary with polarisation, and how accurate this variation can be estimated. The actual level of the InSAR coherence affects the overall performance through the possible estimation accuracy of the observables rather than in a direct way. The variation of InSAR coherence and phase with polarisation and the uncertainty in their estimationas a consequence of nonunity coherence-depend on system parameters as well as on structural properties of the volume scatterer under consideration.

In Section 2, the individual decorrelation contributions induced by the system, the imaging geometry, and the scattering process are discussed with respect to Pol-InSAR volume parameter inversion. The main system parameters that impact the overall interferometric coherence are reviewed. The random volume over ground (RVoG) scattering model is used to describe the effect of the scatterer on the InSAR observables as a function of system parameters. Even if the discussion is held in a more general frame, the main scenario considered in this paper is forest scattering at Lband. Section 3 investigates the achievable performance of a repeat-pass Pol-InSAR mission scenario. For this, two actual L-band missions will be considered as illustrative examples (ALOS/PalSAR and TerraSAR-L). It is shown that the achievable performance will be strongly affected even by moderate levels of temporal decorrelation. Hence, several single-pass Pol-InSAR mission scenarios will be investigated in Section 4. Such systems use multiple satellites flying in close formation and allow for the acquisition of interferometric and polarimetric data during one satellite pass, thereby minimizing the distortions from temporal decorrelation $[22,23,24]$. The performance analysis for a potential TerraSAR-L cartwheel configuration illustrates the excellent Pol-InSAR parameter inversion accuracy to be expected from such a polarimetric single-pass interferometer. Section 5 concludes the paper with a general discussion of the potentials and limitations of the investigated Pol-InSAR mission scenarios for the acquisition of polarimetric and interferometric data on a global scale.

\section{PERFORMANCE ANALYSIS}

In this section, we discuss the major system and scatterer parameters which affect the accuracy of the Pol-InSAR volume parameter inversion. A key quantity in estimating the performance of any interferometric SAR system is coherence. Assuming additive and statistically independent error sources, the total coherence $\tilde{\gamma}_{\text {tot }}$ including both the interferometric correlation coefficient and the interferometric phase is given by the product

$$
\tilde{\gamma}_{\text {tot }}=\gamma_{\mathrm{SNR}} \cdot \gamma_{\mathrm{Quant}} \cdot \gamma_{\mathrm{Amb}} \cdot \gamma_{\mathrm{Coreg}} \cdot \gamma_{\mathrm{Geo}} \cdot \gamma_{\mathrm{Az}} \cdot \tilde{\gamma}_{\mathrm{Vol}} \cdot \tilde{\gamma}_{\mathrm{Temp}},
$$

where the right-hand side describes the individual error contributions:

(i) $\gamma_{\mathrm{SNR}}$ : finite SNR due to thermal noise (scalar contribution),

(ii) $\gamma_{\text {Quant }}$ : quantization errors (scalar contribution),

(iii) $\gamma_{\mathrm{Amb}}$ : range and azimuth ambiguities (scalar contribution),

(iv) $\gamma_{\text {Coreg: coregistration and processing errors (scalar con- }}$ tribution),

(v) $\gamma_{\mathrm{Geo}}$ : baseline decorrelation (scalar contribution),

(vi) $\gamma_{\mathrm{Az}}$ : decorrelation due to Doppler shift (scalar contribution),

(vii) $\tilde{\gamma}_{\text {Vol }}$ : volume decorrelation (complex contribution),

(viii) $\tilde{\gamma}_{\text {Temp }}$ : temporal decorrelation (complex contribution).

The first six terms are decorrelation contributions due to system, processing, and acquisition geometry effects. They are 
scalar quantities as they contribute only to the overall interferometric correlation coefficient. The last two terms are introduced by the scatterer and reflect its structural and temporal stability properties. They are complex contributions as they also affect the measured interferometric phase. In the following, the individual contributions will be discussed.

\subsection{Volume decorrelation}

The penetration into and through natural volume scatterers (such as vegetation, sand, and ice) at longer wavelengths makes volume decorrelation an important decorrelation contribution. At the same time, it is $\tilde{\gamma}_{\text {Vol }}$ that contains the physical information about the vertical structure of the volume scatterer as it is directly related-after range spectral filtering - to the Fourier transform of the vertical distribution of the effective scatterers $\rho_{V}(z)$ as $[4,7,13]$

$$
\tilde{\gamma}_{\text {Vol }}=\frac{\int \rho_{V}\left(z^{\prime}\right) \exp \left(i \kappa_{z} z^{\prime}\right) d z^{\prime}}{\int \rho_{V}\left(z^{\prime}\right) d z^{\prime}}
$$

where $\kappa_{z}$ is the effective vertical interferometric wavenumber after range spectral filtering, which depends on the imaging geometry and the radar wavelength

$$
\kappa_{z}=\frac{\kappa \Delta \theta}{\sin \left(\theta_{\text {inc }}\right)}
$$

with $\kappa=4 \pi / \lambda$ for a repeat-pass mission scenario $(\kappa=2 \pi / \lambda$ for a single-pass mission scenario), the reference incidence angle $\theta_{\text {inc }}$, and the incidence angle difference $\Delta \theta$ between the two interferometric images induced by the baseline. To perform a quantitative evaluation of $\tilde{\gamma}_{\text {Vol }}$, a scattering model describing the vertical distribution of the effective scatterers $\rho_{V}(z)$ has to be introduced. An appropriate model for this is the random volume over ground (RVoG) model $[7,12,13]$ which has been successfully exploited over the last years for quantitative forest parameter estimation from multiparameter InSAR data. The RVoG is a two-layer model (vegetation layer and ground) that expresses the interferometric coherence and phase as a function of four scatterer parameters: (1) the volume thickness that corresponds to vegetation height, (2) the volume extinction coefficient that describes the attenuation through the vegetation layer, (3) the effective groundto-volume amplitude ratio, defined as the ratio of the ground scattering amplitude attenuated by the volume to the volume scattering amplitude, and (4) the phase related to the underlying topography. According to the RVoG model, the complex interferometric coherence $\tilde{\gamma}_{\mathrm{Vol}}(\vec{w})$ after range spectral filtering is given by $[12,13]$

$$
\tilde{\gamma}_{\mathrm{Vol}}(\vec{w})=\exp \left(i \phi_{0}\right) \frac{\tilde{\gamma}_{V}+m(\vec{w})}{1+m(\vec{w})},
$$

where $\vec{w}$ is a unitary vector that defines the polarisation of the interferogram and indicates the polarimetric dependency. $\tilde{\gamma}_{V}$ denotes the coherence for the volume alone, which depends on the extinction coefficient $\sigma$ for the random volume and the volume thickness $h_{V}$ as

$$
\begin{aligned}
\tilde{\gamma}_{V} & =\frac{I}{I_{0}}, \\
I & =\int_{0}^{h_{V}} \exp \left(i \kappa_{z} z^{\prime}\right) \exp \left(\frac{2 \sigma z^{\prime}}{\cos \theta_{0}}\right) d z^{\prime}, \\
I_{0} & =\int_{0}^{h_{V}} \exp \left(\frac{2 \sigma z^{\prime}}{\cos \theta_{0}}\right) d z^{\prime},
\end{aligned}
$$

where $\phi_{0}$ is the phase related to the ground topography, and $m$ is the effective ground-to-volume scattering ratio accounting for the attenuation through the volume

$$
m(\vec{w})=\frac{m_{G}(\vec{w})}{m_{V}(\vec{w}) I_{0}},
$$

where $m_{G}$ is the scattered return from the ground seen through the vegetation (including direct surface and dihedral scattering contributions) and $m_{V}$ is the direct volume scattering return [12].

The extinction coefficient $\sigma$ corresponds to a mean extinction value for the vegetation layer expressing scattering and absorption losses. It is a function of the density of scatterers in the volume and their dielectric constant, and is assumed to be independent of polarisation $[12,13]$. Changes of polarisation influence the interferometric coherence through the variation of the ground-to-volume amplitude ratio $m$ that is the only model parameter that depends on the polarisation of the incident wave $\vec{w}$. In the limit of zero extinction coefficient, $\tilde{\gamma}_{V}$ becomes the well-known $\sin (x) / x$ decorrelation function:

$$
\tilde{\gamma}_{V}=\exp \left(i \phi_{0}+\frac{i \kappa_{z} h_{V}}{2}\right) \frac{\sin \left(\kappa_{z} h_{V} / 2\right)}{\kappa_{z} h_{V} / 2} .
$$

According to (4), the effective phase center is located above the ground at a height that depends on the ground-tovolume amplitude ratio $m$ as well as the attenuation length of the vegetation layer.

Figure 1 shows the variation of the interferometric phase (Figure 1a) and the interferometric coherence (Figure 1b) as predicted by the RVoG model for a volume thickness $h_{V}=$ $20 \mathrm{~m}$, with an extinction coefficient $\sigma=0.3 \mathrm{~dB} / \mathrm{m}$, and an interferometric configuration with a vertical wavenumber of $\kappa_{z}=0.15 \mathrm{rad} / \mathrm{m}$ (corresponding to a $2 \pi$ height of $40 \mathrm{~m}$ ) as a function of the ground-to-volume amplitude ratio $m$ that varies from $-20 \mathrm{~dB}$ to $20 \mathrm{~dB}$. For illustration, we have assumed 16 independent looks in deriving the interferometric phase errors. Looking at the interferometric phase variation (blue continuous line), one can see that the phase center for practically zero ground contribution (at $m=-20 \mathrm{~dB}$ ) is located two thirds of the total volume height (indicated by the red dashed line) above ground (indicated by the blue dashed line) and moves monotonically with increasing ground contribution towards ground level that is reached at $m$ higher than $10 \mathrm{~dB}$. 


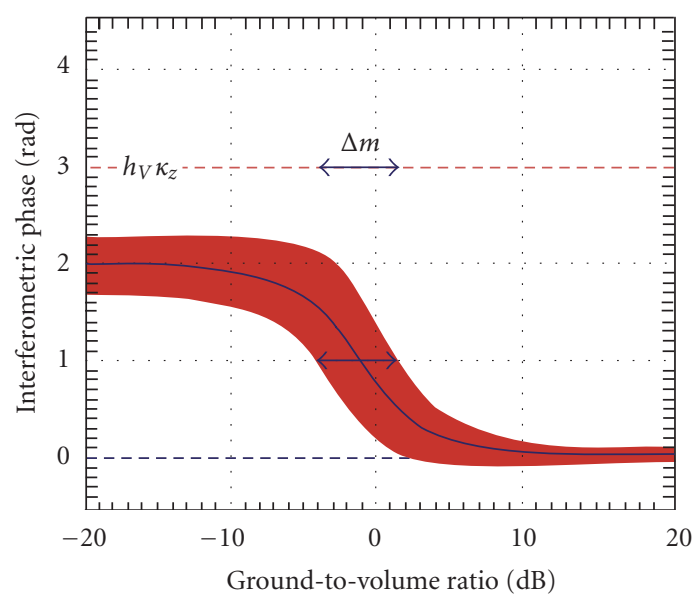

(a)

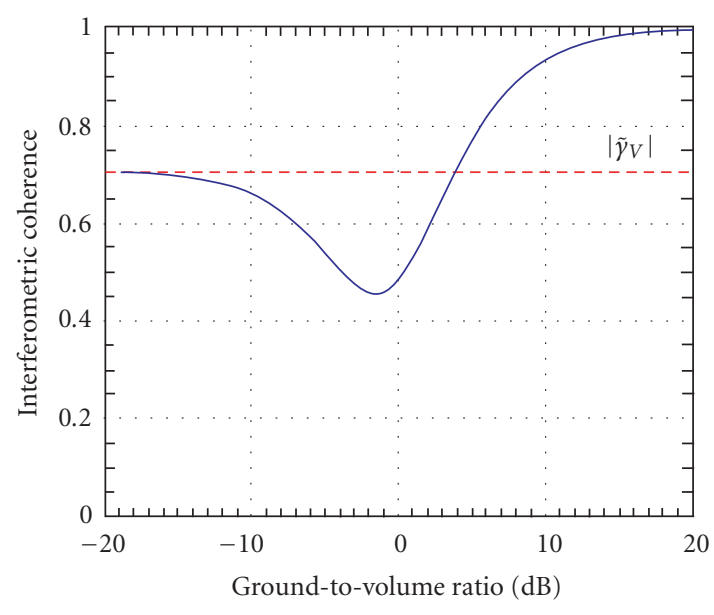

(b)

FIGURE 1: (a) Interferometric phase and (b) interferometric coherence as a function of the ground-to-volume amplitude ratio $m\left(h_{V}=20 \mathrm{~m}\right.$, $\sigma=0.3 \mathrm{~dB} / \mathrm{m}, \kappa_{z}=0.15 \mathrm{rad} / \mathrm{m}, 16$ looks $)$.

In contrast to the phase behaviour, the interferometric coherence variation, shown in Figure $1 \mathrm{~b}$, is not monotonic with $m$ : starting from almost no ground contribution $(m=-20 \mathrm{~dB})$ with a coherence corresponding to the volume layer alone (in this particular case at 0.7 ), the coherence decreases with increasing ground contribution: due to the scattering contribution at the bottom of the volume, the overall (volume + ground) scattering center moves towards ground. This way, the effective volume seen by the interferometer increases, which in turn increases volume decorrelation. Further amplification of the ground contributionbeyond a certain level-leads now to rising coherence values as the ground becomes more and more the dominant scattering contribution, and finally, for very strong ground contributions, the coherence converges to one.

The separation of the phase centers at the different polarisations (i.e., for different values of $m$ ) depends on both the variation of the phase center with $m$ and the standard deviation of the phase estimate associated to the corresponding interferometric coherence value. In the phase variation plot (Figure 1a), the red tube indicates the phase \pm 1 standard deviation region defined by the corresponding coherence variation (Figure $1 \mathrm{~b}$ ) for 16 looks [25, 26, 27]: the standard deviation reaches its maximum in the lower coherence region $(-7 \mathrm{~dB}<m<2 \mathrm{~dB})$ and its minimum in the high coherence area $(m>10 \mathrm{~dB})$.

The ability of a system configuration to separate the phase centers at the different polarisations (for a given scatterer configuration) can be expressed as the amount of the ground-to-volume ratio variation $\Delta m$ required to cause a phase variation larger than the phase standard deviation at a given reference point on the $m$-axis. It becomes obvious that thinner and/or steeper tubes correspond to a better Pol-InSAR performance, as a vertical phase center separation larger than the standard deviation can be achieved by smaller $\Delta m$. Note that in Figure 1 the tube is only due to the effect of volume decorrelation (assuming an ideal InSAR system). Any of the system-induced decorrelation contributions of (1) will further reduce the coherence values leading to a thicker tube. In this sense, system configurations that provide overall thin and steep tubes-and keep the system-induced tube contribution small compared to the volume decorrelation contribution-are better suited for Pol-InSAR applications than broad tubes with small phase variation.

On the other hand, scattering scenarios in which the variation of polarisation leads to a wide range of $m$ values located in the sensitive area of the phase tube allow optimal parameter inversion. The range of $m$ values depends on the strength of the underlying scattering process and its attenuation by the vegetation layer.

\subsubsection{Imaging geometry parameters}

The choice of the spatial baseline has always to be optimised with respect to the individual applications. In conventional DEM generation, for example, large baselines that provide high phase to height sensitivity are desired, limited only by the available system bandwidth (range spectral decorrelation) and the terrain conditions. The baseline requirements are different in the case of Pol-InSAR applications over volume scatterers. Especially at longer wavelengths volume decorrelation dictates the maximum useful baseline length. Figure 2 shows the volume decorrelation according to (5) expected for a volume height of $20 \mathrm{~m}$ as a function of the vertical wavenumber $\kappa_{z}$ for different volume extinction values. The coherence drops below the critical mark of 0.3 for a $\kappa_{z}$ value on the order of $0.2-0.3 \mathrm{rad} / \mathrm{m}$. This corresponds to about $40 \%$ of the critical baseline of ALOS-PalSAR and about $7 \%$ of the critical baseline of TerraSAR-L (assuming $80 \mathrm{MHz}$ bandwidth).

However, note that even if a larger baseline increases volume decorrelation, it still provides a higher phase to height sensitivity that may compensate (up to a certain baseline) the 


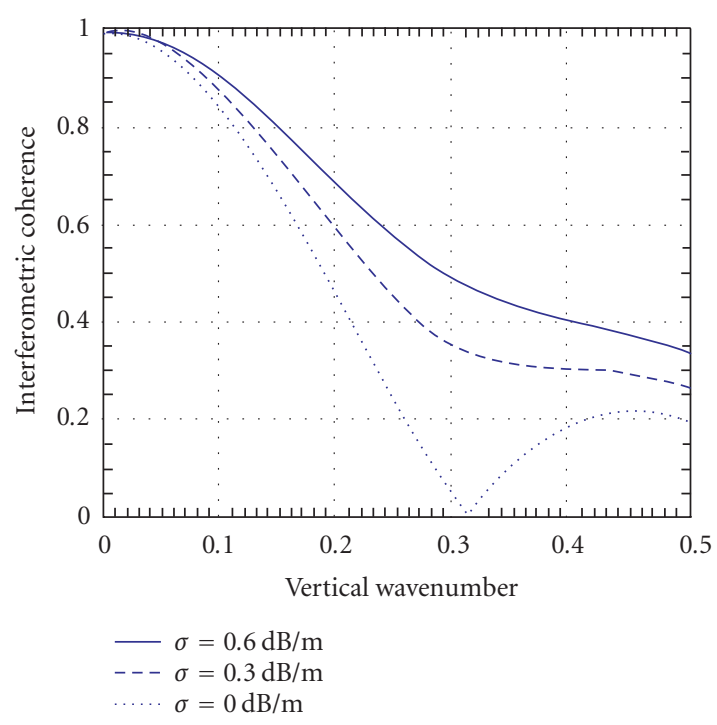

FIGURE 2: Volume decorrelation.

loss in coherence. In other words, the error in height caused by a given phase standard deviation at a small baseline may be finally larger than the error for a larger baseline, even if for the larger baseline the phase standard deviation is larger due to the higher volume decorrelation. The realisation of small baselines, in order to keep volume decorrelation low and to allow high coherence levels, can be a promising concept as long as the system-induced decorrelation effects are kept small. If this is not the case, the system-induced decorrelation becomes large relative to the underlying volume decorrelation and the small baseline concept fails due to the adverse phase-to-height uncertainty transformation. The variation of the Pol-InSAR performance with different baseline lengths will be demonstrated in Sections 3 and 4. As a first rule of thumb, the baselines should be chosen such that the magnitude of the system-induced height errors is comparable to the errors from volume decorrelation.

Another geometric parameter that affects the performance of a Pol-InSAR configuration is the incident angle. In general, an increase of the incident angle leads to a reduction of the backscattered signal and thus to an increase of the SNR decorrelation contribution. According to [28], the variation of backscattering (at X-, C-, and L-band) from forest is in average $3-5 \mathrm{~dB}$ for incident angles in the range between 20 and 50 degrees. This affects-as already discussed-the estimation performance due to the additional decorrelation contribution, and-if not accounted for-introduces a bias in the parameter estimates.

Even more important is the dependency of the ground attenuation on the incident angle. With increasing incident angle, the travelled distance of the transmitted and scattered waves through the vegetation layer increases, thereby increasing the attenuation of the wave and making the effective ground contribution weaker. Figure 3 a shows the wave attenuation as a function of incidence angle assuming a volume height of $h_{V}=20 \mathrm{~m}$ for three different extinction coefficients: $\sigma=0.3,0.6$, and $0.9 \mathrm{~dB} / \mathrm{m}$. It is characteristic that a variation of the incident angle from 20 to 50 degrees has the same effect as an increase of vegetation height from 20 to 30 meters. Assuming that in first order the (polarimetric) dynamic range of the ground scattering is independent of the incident angle, the range of the effective ground-to-volume ratios remains constant with increasing incident angle but is shifted towards lower $m$ values as indicated in Figure 3b. At 35 degrees, the effective ground-to-volume ratios are about $3 \mathrm{~dB}$ lower than at 25-degree incidence-for a mean extinction of $0.6 \mathrm{~dB} / \mathrm{m}$. In this sense, steeper incident angles are favourable as they lead to higher $m$ values. A variation of the (polarimetric) dynamic range of ground scattering with incident angle will additionally affect the performance.

\subsubsection{Reference scenario}

The discussion above makes clear that the performance of a Pol-InSAR system depends strongly on the parameters of the scattering scenario. Data derived from simulations of a Scots pine forest stand [29] will be used to define an appropriate reference for the performance analysis (cf. Table 1). Note that the parameters in [29] have been derived for an incident angle of $45^{\circ}$. In order to be compatible with the incident angle range of the radar sensors considered in Sections 3 and 4 (the maximum incident angle for TerraSAR-L operating in full polarimetric mode is $36^{\circ}$ ), the parameter set had to be adapted appropriately. The major difference relates to the lower incident angle that will increase the ground-to-volume amplitude ratios where a shift of the $m$ values by $+5 \mathrm{~dB}$ has been assumed. This increase seems to be justified by the lower total extinction in the volume, the stronger ground contributions for steeper incident angles, and the fact that the vegetation model in [29] did not account for the scattering from understory. Furthermore, equal scattering coefficients of $-11 \mathrm{~dB} \mathrm{~m} / \mathrm{m}^{2}$ will be assumed for all polarisations. By this, we avoid different SNR values that would lead to different performance predictions for the different polarisations. Note that the number of independent radar pulses recorded in the cross-polar channel is twice the number of pulses recorded in each copolar channel. This increase of independent samples corresponds to an improvement of the SNR by $3 \mathrm{~dB}$ which compensates in part the lower scattering in cross-polarisation. The chosen scattering coefficient corresponds hence to an effective cross-polar scattering coefficient of $-14 \mathrm{~dB} \mathrm{~m} \mathrm{~m}^{2} / \mathrm{m}^{2}$ which we regard as a lower bound for the strength of the scattered signal. Hence, the parameters provided in Table 1 reflect rather conventional assumptions for the investigated Scots pine forest scenario.

\subsection{Temporal decorrelation}

Temporal decorrelation is probably the most critical factor for a successful implementation of Pol-InSAR parameter inversion techniques in terms of conventional repeat-pass InSAR scenarios. Similar to any other system-induced decorrelation contribution, temporal decorrelation reduces the performance of a Pol-InSAR configuration by biasing the volume decorrelation contribution that is used for parameter 


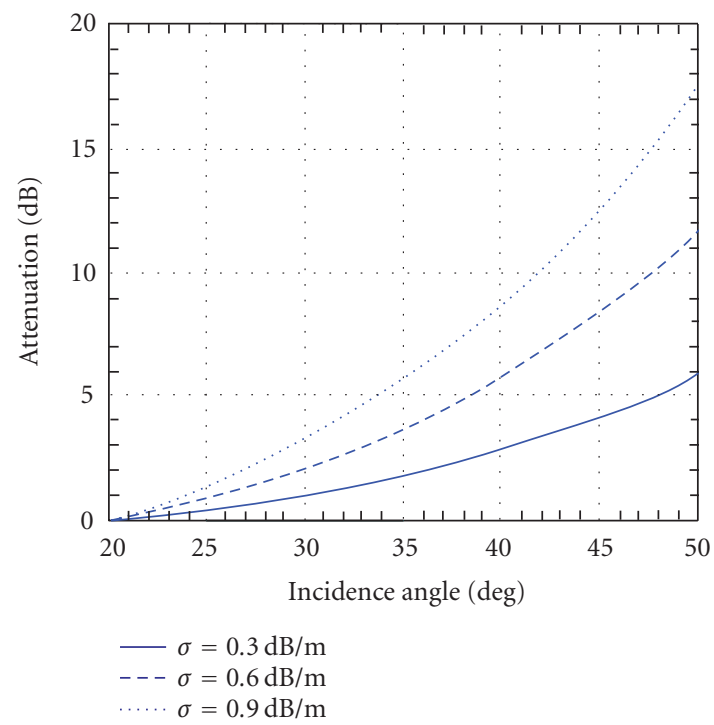

(a)

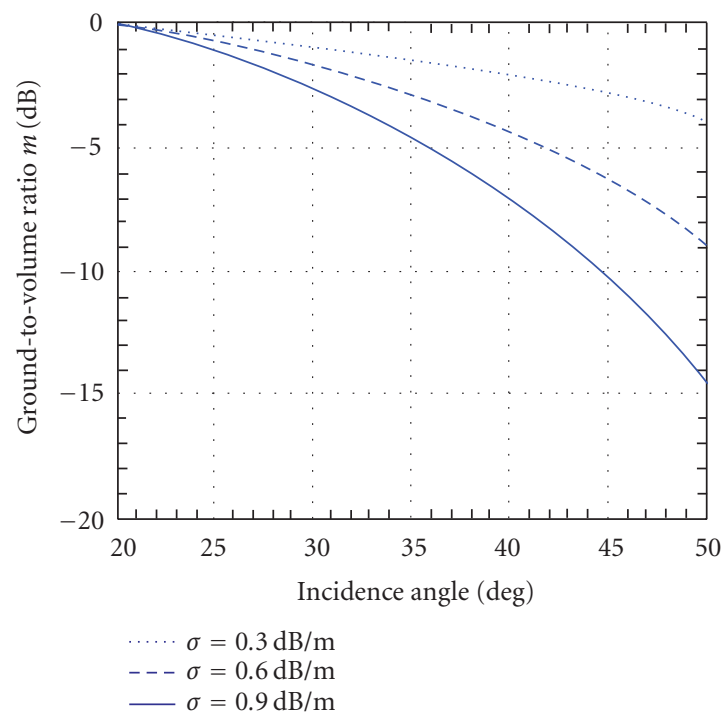

(b)

FIGURE 3: (a) Wave attenuation and (b) variation of ground-to-volume ratio as a function of the incident angle (volume height: 20 m; extinction coefficient: $0.3,0.6$, and $0.9 \mathrm{~dB} / \mathrm{m})$.

TABle 1: Parameters of reference scenario.

\begin{tabular}{lc}
\hline Reference scenario & $\begin{array}{r}\text { Scots pine forest with a stem density } \\
\text { of } 0.055 \mathrm{stems} / \mathrm{m}^{2}\end{array}$ \\
\hline Scattering coefficient & $\begin{array}{r}\sigma_{0}>-11 \mathrm{~dB} \mathrm{~m} \mathrm{~m}^{2} / \mathrm{m}^{2} \text { (copolarisation) } \\
\sigma_{0}>-14 \mathrm{~dB} \mathrm{~m}^{2} / \mathrm{m}^{2} \text { (cross-polarisation) }\end{array}$ \\
Incident angle & $<35^{\circ}$ \\
Extinction & $0.3 \mathrm{~dB} / \mathrm{m}$ \\
Ground-to-volume ratios & $-26 \mathrm{~dB}<m<-2 \mathrm{~dB}$ \\
Height & $20 \mathrm{~m}$ \\
\hline
\end{tabular}

inversion. This leads to a larger standard deviation of the InSAR phase-for the same number of looks - and increases the error bars of the parameter estimates.

Regarding vegetation height inversion applications, the effect of a biased volume decorrelation is more important as it leads to overestimated heights. Figure $4 \mathrm{a}$ shows the estimation error (in first order according to the RVoG model) for a volume height of $20 \mathrm{~m}$ (assuming $\sigma=0.0 \mathrm{~dB} / \mathrm{m}$ ) as a function of temporal decorrelation at three vertical wavenumbers $\left(\kappa_{z}=0.1,0.15\right.$, and $\left.0.2 \mathrm{rad} / \mathrm{m}\right)$. The corresponding coherence variations are shown on the right-hand side. One can see that, for small baselines (i.e., $\kappa_{z}=0.1 \mathrm{rad} / \mathrm{m}$ ), temporal decorrelation on the order of 0.9 already causes an error on the order of $20 \%$. The impact becomes weaker for larger baselines-as a consequence of an increased volume decorrelation. For the 0.2 vertical wavenumber case, the height bias reaches $10 \%$ for a volume decorrelation level of about 0.7 . This makes clear that larger spatial baselines are advantageous in the presence of moderate temporal decorrelation as they minimise the introduced bias. However, larger baselines lead at the same time to lower overall coherence values and become critical at high temporal decorrelation levels.

In order to compensate the degradation in estimation performance, temporal decorrelation has to be accounted for in the modelling/inversion methodology. The amount of temporal decorrelation for a given observation time depends on the changing processes occurring. Unfortunately, common natural decorrelation processes, as wind, evapotranspiration, thawing, and freezing processes, rain and snow events, as well as human activities appear in time stochastic (or with very short correlation time) rather than in a regular fashion. Thus, even if it is possible to relate (or to model) the decorrelation caused by different changing processes, it is very difficult to conclude about the decorrelation rising within a given time interval. Except for seasonal, natural, and cultivation cycles, geographic-dependent weather event statistics and distribution statistics, as well as the reaction time of the scatterers to certain weather phenomena, there are not many constants that will allow even a rough assessment of the amount of temporal decorrelation. Thus, there are no models that are able to predict in a realistic way general temporal decorrelation effects as a function of time.

Hence, temporal decorrelation effects-in the absence of detailed knowledge about the changing processes-can only be incorporated in scattering models in a very abstract way $[30,31]$. For long temporal baselines, both the volume and the ground scattering components may be affected by temporal decorrelation and the decorrelation coefficient may even become complex-introducing a phase bias. In this case, inversion performance collapses, as the number of unknowns becomes larger than the number of available observables. However, even if the general temporal decorrelation scenario cannot be accounted for, special cases of dynamic processes 


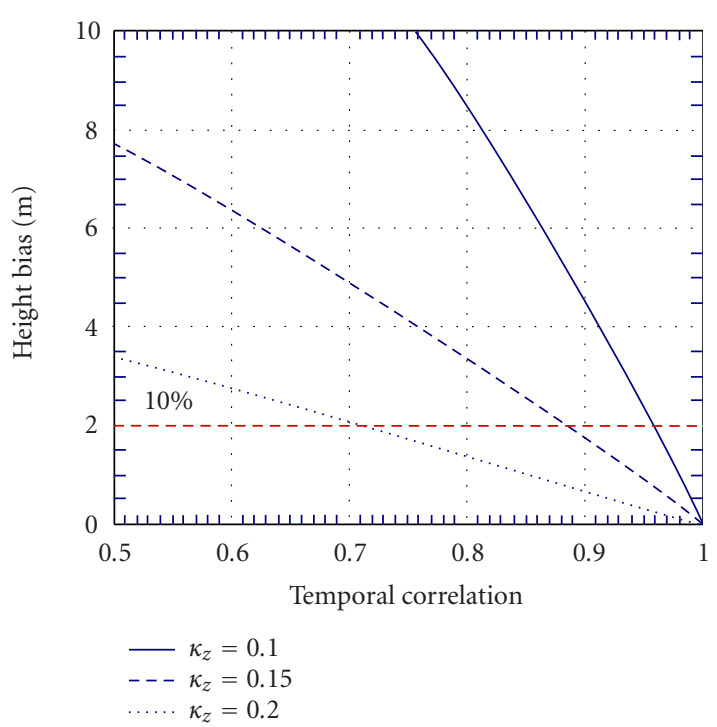

(a)

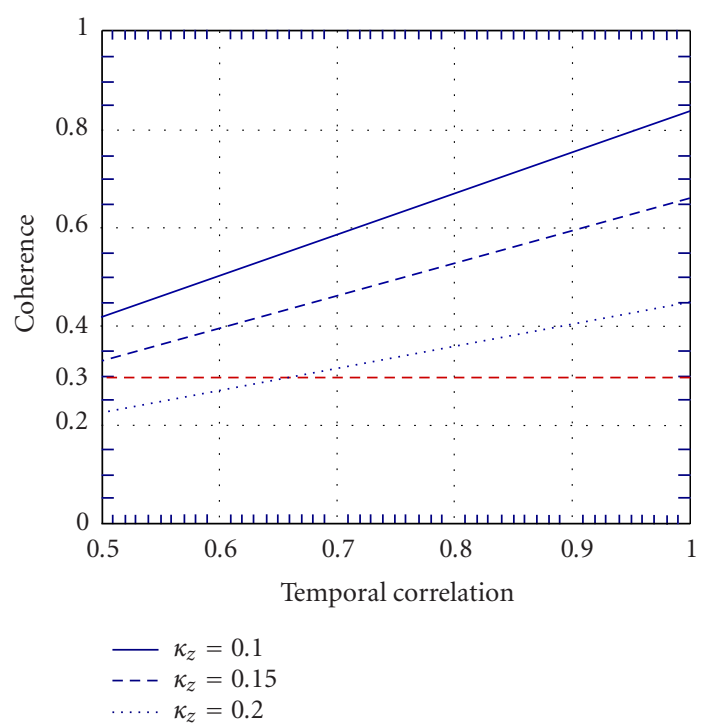

(b)

FIgURE 4: (a)Height bias and (b) interferometric coherence as a function of temporal decorrelation.

may be accounted under certain assumptions [30]. With decreasing temporal baseline, the most common temporal decorrelation effect over forested terrain is wind-induced movement of "unstable" scatterers within the canopy layer as, for example, leaves and/or branches, and so forth. This leads to a relative change in the positions of the effective scatterers inside the resolution cell in the two acquisitions. According to this decorrelation scenario only the volume layer is affected by temporal decorrelation and can be accounted for in the model by a scalar decorrelation coefficient. This case can now be inverted

(i) in terms of a single baseline by fixing the extinction coefficient, leading to biased volume height estimates [30] or

(ii) in terms of a dual-baseline Pol-InSAR scenario without any additional assumptions and with enhanced inversion performance [31,32].

Regarding design and operation of a repeat-pass spaceborne InSAR mission, the definition of the repeat cycle has a critical impact as it affects a wide range of important issues such as land coverage, mission duration, fuel consumption, and so forth. The prevalent conclusion that the minimum repeatpass time interval leads to an optimum temporal decorrelation performance becomes controvertible under the light of a quasistochastic temporal decorrelation behaviour, especially for scenarios with short repeat-pass times on the order of a few days. Today, there is not sufficient evidence that allows to conclude about if-for example - a three-day repeat-pass mission scenario provides an appreciably better performance on a global scale (with respect to quantitative parameter estimation) than a six-day or one-week repeat-pass time scenario. On the other hand, a twice as long repeat-pass time significantly relaxes mission and operation constraints.

\subsection{Thermal noise decorrelation}

The finite radiometric sensitivity of each interferometric channel will cause a coherence loss $\gamma_{\mathrm{SNR}}$ which is given by $[33,34]$

$$
\gamma_{\mathrm{SNR}}=\frac{1}{1+\mathrm{SNR}^{-1}}
$$

with the signal-to-noise ratio

$$
\mathrm{SNR}=\frac{\sigma_{0}\left(\theta_{\text {inc }}-\alpha\right)}{\operatorname{NESZ}\left(\theta_{\text {inc }}-\alpha\right)}
$$

where $\sigma_{0}$ is the normalised backscattering coefficient. NESZ is the noise equivalent sigma zero level of the system which can be derived as $[35,36]$

$$
\mathrm{NESZ}=\frac{4^{4} \pi^{3} r^{3} v \sin \left(\theta_{\mathrm{inc}}-\alpha\right) k T B_{r g} F L}{P_{\mathrm{Tx}} G_{\mathrm{Tx}} G_{\mathrm{Rx}} \lambda^{3} c_{0} \tau_{p} \mathrm{PRF}}
$$

with slant range $r$, satellite velocity $v$, incident angle $\theta_{\text {inc }}$, local slope angle $\alpha$, Boltzmann constant $k$, bandwidth of the radar pulse $B_{r g}$, noise figure $F$, losses $L$, transmit power $P_{\mathrm{Tx}}$, gain of the transmit and receive antennas $G_{\mathrm{Tx}}$ and $G_{\mathrm{Rx}}$, wavelength $\lambda$, velocity of light $c_{0}$, pulse duration $\tau_{p}$, and pulse repetition frequency PRF. The gain of the antenna can be approximated by [35]

$$
G_{\{\mathrm{Tx}, \mathrm{Rx}\}}=\frac{4 \pi}{\lambda^{2}} A_{\{\mathrm{Tx}, \mathrm{Rx}\}},
$$

where $A_{\mathrm{Tx}}$ and $A_{\mathrm{Rx}}$ are the antenna areas of the transmitter and receiver, respectively. 


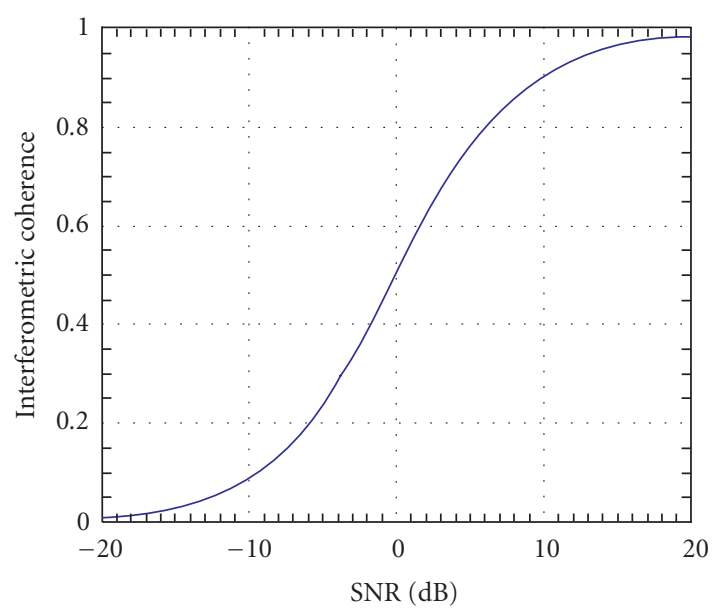

FIGURE 5: SNR decorrelation.

For a fixed NESZ, $\gamma_{\mathrm{SNR}}$ depends on the backscattered intensity and is therefore a function of frequency, polarisation, and incidence angle. In Figure 5, $\gamma_{\mathrm{SNR}}$ is plotted against SNR. For an SNR of $0 \mathrm{~dB}, \gamma_{\mathrm{SNR}}$ becomes 0.5 , for SNR values below $-10 \mathrm{~dB} \gamma_{\mathrm{SNR}}$ drops below 0.1, and for SNR values above $15 \mathrm{~dB} \gamma_{\mathrm{SNR}} \sim 1$. In consequence, for SAR systems characterised by NESZ values on the order of $-25 \mathrm{~dB}$ to $-30 \mathrm{~dB}$, SNR decorrelation over vegetated scatterers can be-in general-neglected. However, for surface scatterersespecially at longer wavelengths-characterised by very low backscattering, it becomes an issue.

Regarding now the effect on vegetation height inversion, and ignoring for the moment all other decorrelation contributions, SNR decorrelation superimposes to volume decorrelation, and reduces the overall coherence. This leads-if not accounted for-to an overestimation of volume height, as $h_{V}=f\left(\gamma_{V}\right)<h_{V}=f\left(\gamma_{V} \gamma_{\mathrm{SNR}}\right)$. In order to provide a feeling for the amount of overestimation to be expected for a volume height of $20 \mathrm{~m}$, the height error $h_{V}\left(\gamma_{V} \gamma_{\mathrm{SNR}}\right)-h_{V}\left(\gamma_{V}\right)$ is plotted in Figure 6a as a function of the backscattering coefficient $\sigma_{0}$ for a fixed NESZ of $-25 \mathrm{~dB}$ at three vertical wavenumbers $\left(\kappa_{z}=0.1,0.15\right.$, and $\left.0.2 \mathrm{rad} / \mathrm{m}\right)$. One can see that the $10 \%$ height error level (indicated by the red dashed line) is reached for the shorter baseline (i.e., $\kappa_{z}=0.1 \mathrm{rad} / \mathrm{m}$ ) for $\sigma_{0}$ values of about $-11 \mathrm{~dB}$ and for the longer baseline $\left(\kappa_{z}=0.2 \mathrm{rad} / \mathrm{m}\right)$ for $\sigma_{0}$ values of about $-26 \mathrm{~dB}$. These values are on the order of the average $\sigma_{0}$ values expected at Cand L-band over vegetation for all polarisations and for incidence angles between 20 and 40 degrees [26] so that in this case SNR decorrelation is not a critical issue. The variation of the corresponding overall interferometric coherence $\gamma=\gamma_{V} \gamma_{\mathrm{SNR}}$ is also shown in Figure 6b: the coherence values at the right-hand side of each plot (i.e., at $\sigma_{0}=0 \mathrm{~dB}$ ) correspond pretty much to the "pure" volume decorrelation values and drop down with decreasing $\sigma_{0}$ values. The dashed line indicates the 0.3 interferometric coherence level, as a critical level below which quantitative InSAR applications reach the limits of conventional interferometric performance. As seen, this level is reached for $\sigma_{0}$ values lower than $-20 \mathrm{~dB}$, that is, it is in general not critical for forest applications. Nevertheless, weak SNR effects (for systems with NESZ levels better than $-25 \mathrm{~dB}$ ) become important (and critical) at short baseline configurations when the overall expected coherence (including the volume scatterer and the system) reaches high levels-due to the unfavourable phase to height scaling.

\subsection{Quantization}

Another potential error source is due to the quantization of the recorded raw data signals $[36,37]$. In a strict sense, quantization errors have to be regarded as a nonlinear and signaldependent signal distortion, but for the current investigation it is reasonable to approximate them as additive white noise. This is justified by comparing the phase error estimates computed from the signal-to-quantization noise ratio (SQNR) to the phase errors obtained from a simulation of the complete quantizer (cf. Table 2). For this simulation, a nonuniform Lloyd-Max quantizer [38] has been used, which will minimize the distortion for a given bit rate in case of a Gaussian signal (assuming independent Cartesian quantization of I and Q channels, see also [39]). It becomes clear that quantization could bias the Pol-InSAR performance in case of a low bit rate. Hence, a quantization with $4+4$ bits/sample will be assumed in the following. This will lead to a signal-toquantisation noise ratio (SQNR) of $20.2 \mathrm{~dB}$ and a coherence of $\gamma_{\text {Quant }}=0.991$.

\subsection{Coregistration errors}

Processing and coregistration errors can be modelled as phase aberrations in the transfer functions of the SAR processor. With $\delta_{a z}$ and $\delta_{r g}$ being the relative azimuth and range shift between the two interferometric images in fractions of a resolution cell, the coherence loss due to misregistration is given by [34]

$$
\gamma_{\text {Coreg }}=\frac{\sin \left(\pi \delta_{r g}\right)}{\pi \delta_{r g}} \cdot \frac{\sin \left(\pi \delta_{a z}\right)}{\pi \delta_{a z}} .
$$

A coregistration accuracy of $1 / 10$ of an image pixel can be expected in both azimuth and range. This will yield a coherence of $\gamma_{\text {Coreg }}=0.97$. Such a coherence loss may cause a small height bias during the Pol-InSAR parameter inversion in case of short interferometric baselines (cf. Figure 4a).

\subsection{Ambiguities}

Range and azimuth ambiguities deserve special attention in the case of fully polarimetric SAR systems. This is due to the fact that the acquisition of a fully polarimetric raw data set will require the use of alternating transmit polarisations in order to acquire the full scattering matrix, thereby reducing the effective PRF for the like-polarised components by a factor of two. The rise of azimuth ambiguities could in principle be avoided by a doubling of the PRF but this would in turn cause a significant increase of range ambiguities. Usually, a compromise will be made which includes (1) a reduction of the imaged swath and (2) a rise of the PRF such that both range and azimuth ambiguities have a 


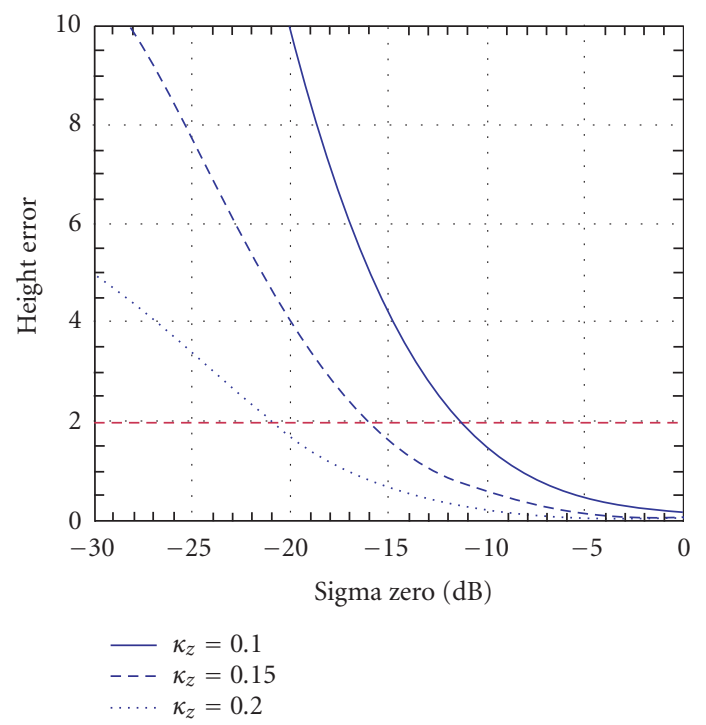

(a)

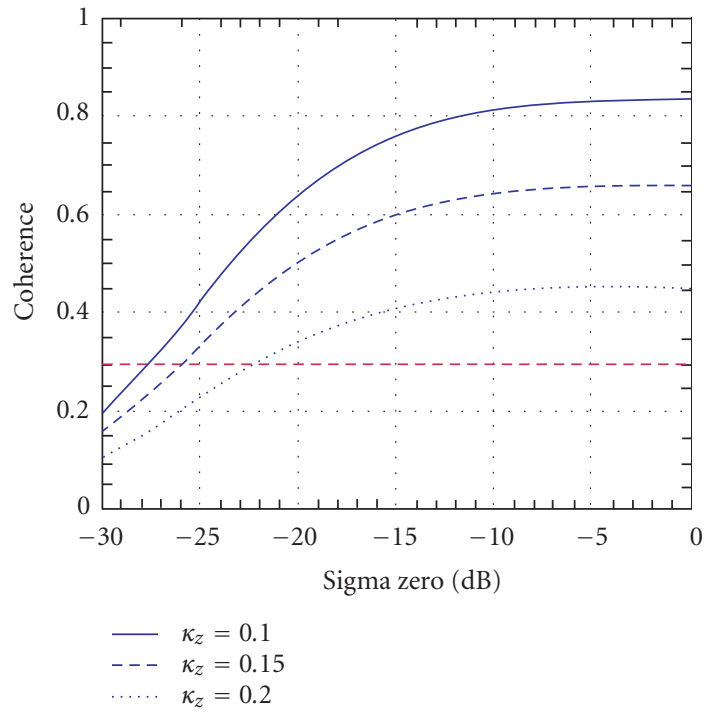

(b)

Figure 6: (a) Height error and (b) interferometric coherence as a function of $\sigma_{0}$ for a fixed NESZ of $-25 \mathrm{~dB}$.

TABLE 2: Signal-to-quantization noise and estimated standard deviation of single channel phase errors for a BAQ with nonuniform LloydMax quantization.

\begin{tabular}{|c|c|c|c|c|c|}
\hline \multirow{2}{*}{ Bits } & \multirow{2}{*}{$\begin{array}{c}\text { SQNR } \\
\text { (Llyod-Max } \\
\text { quantization) }\end{array}$} & \multirow{2}{*}{$\begin{array}{l}\text { Coherence } \\
\text { (theoretic) }\end{array}$} & \multirow{2}{*}{$\begin{array}{c}\text { Interferometric } \\
\text { phase error (from } \\
\text { coherence) }\end{array}$} & \multicolumn{2}{|c|}{$\begin{array}{l}\text { Simulation for optimum } \\
\text { Llyod-Max quantization }\end{array}$} \\
\hline & & & & ( 1 channel) & ( 2 channels) \\
\hline $2+2$ & $9.3 \mathrm{~dB}$ & 0.895 & $40.3^{\circ}$ & $30.7^{\circ}$ & $43.4^{\circ}$ \\
\hline $3+3$ & $14.6 \mathrm{~dB}$ & 0.966 & $23.9^{\circ}$ & $18.8^{\circ}$ & $26.6^{\circ}$ \\
\hline $4+4$ & $20.2 \mathrm{~dB}$ & 0.991 & $13.9^{\circ}$ & $10.5^{\circ}$ & $14.8^{\circ}$ \\
\hline $5+5$ & $26.0 \mathrm{~dB}$ & 0.997 & $7.8^{\circ}$ & $6.1^{\circ}$ & $8.6^{\circ}$ \\
\hline
\end{tabular}

comparable level. Any detailed analysis of the range and azimuth ambiguities will hence strongly depend on the chosen system parameters like PRF, antenna tapering, swath width, incident angles, as well as on the scattering characteristics of the imaged scene. However, many of these parameters have not been specified yet for the systems that will be considered in Sections 3 and 4. It would hence be difficult to make an exact prediction about the ambiguity to signal ratio (ASR) at the current stage of analysis. Reasonable values will be ASR $<-20 \mathrm{~dB}$ for the repeat-pass mission scenarios in Section 3 and ASR $<-14 \mathrm{~dB}$ for the single-pass mission scenarios in Section 4. These values are supported by the TerraSAR-L and ALOS system specifications as well as by detailed performance investigations of a potential TerraSAR-L cartwheel constellation [23, 40, 41]. As argued in [22], ambiguities will combine incoherently in the final interferogram. The question of how far strong ambiguities may also bias the Pol-InSAR parameter inversion accuracy due to a spatially correlated interferometric phase offset clearly deserves further in-depth investigation, which is beyond the scope of this paper. Hence, a simplified model will be adopted which treats the contribution from ambiguities as additional noise with an associated coherence loss given by [33]

$$
\gamma_{\mathrm{Amb}}=\frac{1}{1+\mathrm{RASR}} \cdot \frac{1}{1+\mathrm{AASR}},
$$

where RASR and AASR are the range and azimuth ambiguity to signal ratios, respectively. The coherence loss due to ambiguities would hence be $\gamma_{\mathrm{Amb}}=0.98$ for the repeat-pass mission scenarios and $\gamma_{\mathrm{Amb}}=0.92$ for the single-pass mission scenarios.

\subsection{Baseline and Doppler decorrelation}

The coherence loss from nonoverlapping Doppler and ground range spectra can be compensated performing range and azimuth spectral filtering to a common frequency band $\left(\gamma_{\mathrm{Geo}}=\gamma_{\mathrm{Az}}=1.0\right)$. The reduced bandwidth will imply a reduced number of looks for a given independent postspacing, which will be taken into account in the computation of the final height errors. 


\subsection{Estimation of interferometric phase errors}

From the total coherence $\gamma_{\text {tot }}$, it is now possible to derive the interferometric phase error. This estimation is based on the assumption that all noise contributions to the two interferometric channels may be modelled by a linear superposition of mutually uncorrelated, complex, circular, stationary, white Gaussian processes [34]. The probability density functions of the phase difference $p_{\varphi}(\varphi)$ between the two interferometric SAR channels is then given by [27]

$$
\begin{aligned}
p_{\varphi}(\varphi)= & \frac{\Gamma(n+1 / 2)\left(1-\gamma_{\mathrm{tot}}^{2}\right)^{n} \gamma_{\mathrm{tot}} \cos \varphi}{2 \sqrt{\pi} \Gamma(n)\left(1-\gamma_{\mathrm{tot}}^{2} \cos ^{2} \varphi\right)^{n+1 / 2}} \\
& +\frac{\left(1-\gamma_{\mathrm{tot}}^{2}\right)^{n}}{2 \pi} F\left(n, 1 ; \frac{1}{2} ; \gamma_{\mathrm{tot}}^{2} \cos ^{2} \varphi\right),
\end{aligned}
$$

where $n$ is the number of independent looks, $F$ the Gauss hypergeometric function [42], and $\Gamma$ the gamma function. In order to compute the number of looks, we have to take into account that range and azimuth filtering has been assumed for an optimisation of the interferometric coherence. As a result, the bandwidth in each channel will be reduced, thereby increasing the geometric resolution and decreasing the number of independent looks for a given postspacing. The number of independent looks is given by

$$
n=\frac{\Delta x}{\Delta r g} \cdot \frac{\Delta y}{\Delta a z}
$$

where $\Delta x$ and $\Delta y$ are the independent post spacings of the final Pol-InSAR product in range and azimuth, respectively. The range resolution $\Delta r g$ may be computed from [36]

$$
\Delta r g=\frac{c_{0} \cos (\alpha)}{2 B_{r g} \sin \left(\theta_{\text {inc }}-\alpha\right)} \cdot \frac{B_{\perp, \text { crit }}}{B_{\perp, \text { crit }}-B_{\perp}},
$$

where $B_{\perp}$ is the interferometric baseline perpendicular to the line of sight and

$$
B_{\perp, \mathrm{crit}}=\frac{q B_{r g} \lambda r \tan \left(\theta_{\mathrm{inc}}-\alpha\right)}{c_{0}}
$$

is the critical baseline for the investigated configuration. The factor $q$ will be 1 for a repeat-pass scenario and 2 for a singlepass scenario.

As mentioned above, we assume also azimuth filtering of the two channels prior to forming the interferogram in order to prevent any decorrelation due to different Doppler centroids. In case of a single-pass scenario, this will lead to a degraded azimuth resolution [36]

$$
\Delta a z=\frac{v_{g r d}}{B_{\text {proc }}-\Delta f} \quad \text { with } \Delta f=\left|f_{\mathrm{Dop}, 1}-f_{\mathrm{Dop}, 2}\right|,
$$

where $B_{\text {proc }}$ is the processed Doppler bandwidth $(1200 \mathrm{~Hz}$ for the investigated multistatic TerraSAR-L Pol-InSAR configuration in Section 4). It is clear that the relative shift of the
Doppler centroids will strongly depend on the selected orbital configuration. For a repeat-pass scenario, the Doppler shift due to antenna pointing inaccuracies may be neglected and the azimuth resolution is approximated by

$$
\Delta a z=\frac{v}{B_{\text {proc }}} \approx \frac{d_{\text {ant }}}{2 \cdot 0.888} .
$$

For an estimate of the final phase difference in the complex interferogram, we use the standard deviation of $p_{\varphi}(\varphi)$ from (14) which is given by

$$
\sigma_{\varphi}=\sqrt{\int_{-\pi}^{\pi} \varphi^{2} p_{\varphi}(\varphi) \cdot d \varphi}
$$

The height error is then derived from the interferometric phase error by [43]

$$
\Delta h=\frac{\lambda r \sin \left(\theta_{\text {inc }}\right)}{q 2 \pi B_{\perp}} \cdot \sigma_{\varphi}=\frac{1}{\kappa_{z}} \cdot \sigma_{\varphi} .
$$

Note that the conventional estimation of the interferometric coherence is biased [25] especially for low coherence values. In order to avoid biased coherence estimates, a sufficiently large number of samples has to be used for the coherence estimation. Uncompensated coherence bias leads to an overestimation of the underlying coherence and may lead to an underestimation of the estimated forest height.

Apart from spectral decorrelation, the range bandwidth also affects the SNR decorrelation contribution as it defines the additive thermal noise power contribution. Under the assumption of a constant power spectral density of the thermal noise, the relation between system bandwidth and thermal noise power is linear [35], so that a duplication of system bandwidth leads to a duplication of the noise power thus increasing SNR decorrelation and decreasing the overall coherence. On the other hand, a larger bandwidth offers the advantage of a higher spatial resolution that allows-for achieving the same final resolution - the implementation of a higher number of looks in InSAR processing. The strong decrease of the interferometric phase standard deviation with increasing number of looks is-especially for small look numbersessential for achieving high phase accuracy.

To provide a quantitative assessment of the effect of range bandwidth on the performance of a given Pol-InSAR configuration, the standard deviation of the interferometric phase as a function of system bandwidth is evaluated, accounting the counteraction of SNR decorrelation and the number of available looks. Assuming a linearly increasing noise power and an SNR level of $10 \mathrm{~dB}$ at $50 \mathrm{MHz}$ (typical for a TerraSAR-L-like configuration), Figure 7 shows the variation of the standard deviation of the InSAR phase as a function of the available bandwidth assuming a volume coherence of 0.85 (Figure 7c), 0.70 (Figure 7b), and 0.55 (Figure 7a) (corresponding to a volume height of $20 \mathrm{~m}$ with an extinction coefficient of $0.3 \mathrm{~dB} / \mathrm{m}$ seen by an interferometric system with a vertical wavenumber of $0.1,0.15$, and $0.20 \mathrm{rad} / \mathrm{m}$ at 


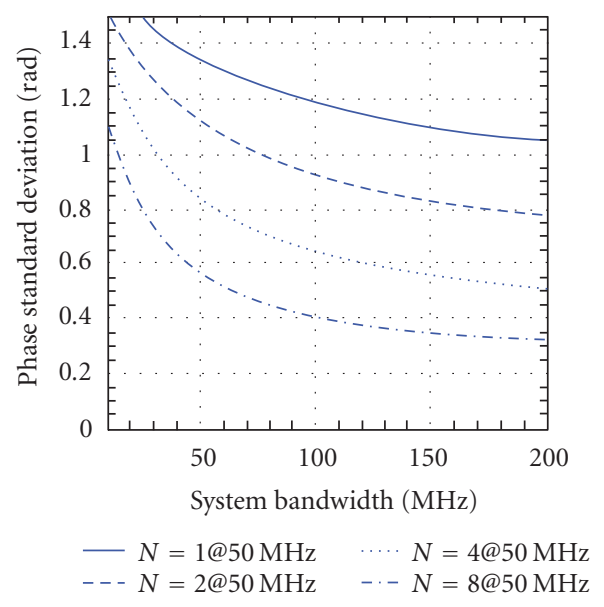

(a)

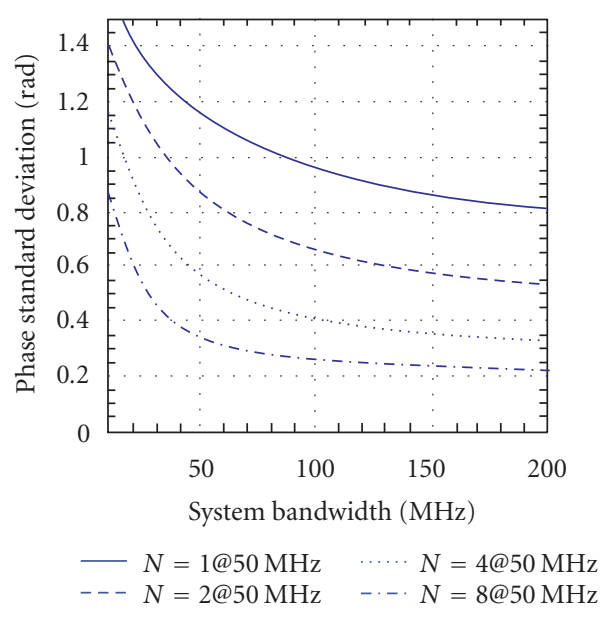

(b)

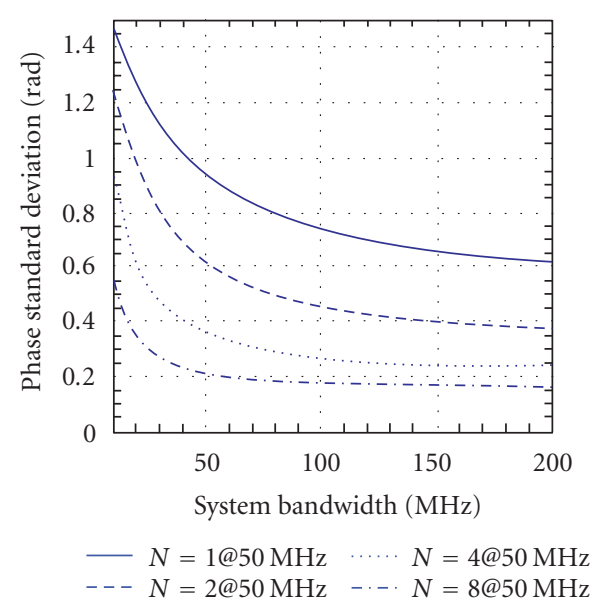

(c)

FIgURE 7: Standard deviation of the InSAR phase as a function of system bandwidth for a volume coherence of (a) 0.55 , (b) 0.70 , and (c) 0.85 . $35^{\circ}$ incidence). With decreasing coherence level, the performance improvement with bandwidth saturates later, because the reduction of phase variation with increasing number of looks is stronger at lower than at higher coherence values. This is a noteworthy difference to the situation given in conventional InSAR topography estimation where-due to the generally high assumed overall coherence levels-the benefit of an increase in bandwidth saturates very fast.

\section{REPEAT-PASS MISSION SCENARIOS}

This section investigates the achievable performance of repeat-pass Pol-InSAR mission scenarios where the polarimetric and interferometric data are acquired with a single satellite. The use of subsequent satellite passes for PolInSAR data collection will imply a significant time-lag of several days between the acquisitions of the two interferometric SAR images. As already mentioned in the introduction, two satellites with fully polarimetric capabilities have been chosen as representative examples for the performance analysis: TerraSAR-L [41] and ALOS/PalSAR [44].

\subsection{TerraSAR-L}

This section analyses the achievable Pol-InSAR performance for TerraSAR-L. A description of the TerraSAR-L mission may be found in $[41,45]$ and the middle column of Table 3 summarises those parameters which have been used in the current performance evaluation. To avoid the necessity of computing the performance for each swath position, a constant antenna loss factor of $3 \mathrm{~dB}$ has been assumed to account for the differences in the antenna gain for different elevation angles. This value seems to be reasonable for the assumed $40 \mathrm{~km}$ swath but the exact range profile will of course depend on the imaged swath width, the incident angle, as well as the selected antenna tapering. The chirp bandwidth of $80 \mathrm{MHz}$ has been chosen to provide a large number of looks for a given range resolution, thereby minimizing the phase errors due to volume, SNR, and temporal decorrelation.

The estimated height errors for TerraSAR-L are shown in Figure 8 as a function of the ground-to-volume scattering ratio $m$ for four different baselines. All errors are indicated as $\pm \sigma_{h}$ (standard deviation of the height error) relative to the mean height of the phase centre. An independent post spacing of $50 \mathrm{~m} \times 50 \mathrm{~m}$ (i.e., $1 / 4 \mathrm{ha}$ ) has been assumed which corresponds to approximately 117 independent looks for the given range bandwidth of $80 \mathrm{MHz}$. The green tubes show the height errors due to volume decorrelation for a vegetation layer with a height of $20 \mathrm{~m}$ and an extinction coefficient of $0.3 \mathrm{~dB} / \mathrm{m}$. The blue areas show additional errors due to the limited system accuracy, and the red areas indicate the total errors in case of temporal decorrelation (solid: $\gamma_{\mathrm{tmp}}=0.8$, dashed: $\gamma_{\mathrm{tmp}}=0.6$, dotted: $\gamma_{\mathrm{tmp}}=0.4$ ). The expected range of ground-to-volume ratios provided in Table 1 $\left(m_{\min }=-26 \mathrm{~dB}\right.$ and $\left.m_{\max }=-2 \mathrm{~dB}\right)$ is indicated by the darker areas of the height error tubes.

It becomes clear that a separation of the phase centres with different polarisations may become difficult for the 
TABle 3: Parameters for performance analysis.

\begin{tabular}{lcc}
\hline Parameter & TerraSAR-L & ALOS/PalSAR \\
\hline Wavelength & $0.238 \mathrm{~m}$ & $0.236 \mathrm{~m}$ \\
Orbit height & $629 \mathrm{~km}$ & $691 \mathrm{~km}$ \\
Chirp bandwidth & $80 \mathrm{MHz}$ & $14 \mathrm{MHz}$ \\
Effective peak Tx power & $4,7 \mathrm{~kW}$ & $2 \mathrm{~kW}$ \\
Duty cycle (for each polarisation) & $3,5 \%(7 \% / 2)$ & $3,5 \%(7 \% / 2)$ \\
Receiver noise figure & $2.5 \mathrm{~dB}$ & $4 \mathrm{~dB}$ \\
Losses (Rx, proc., atm.) & $2 \mathrm{~dB}$ & $2 \mathrm{~dB}$ \\
Losses across swath (40 km swath) & $<3 \mathrm{~dB}$ & $<3 \mathrm{~dB}$ \\
Antenna size (Tx, Rx) & $11 \mathrm{~m} \times 2.86 \mathrm{~m}$ & $1 / 10 \mathrm{pixel} \times 3.1 \mathrm{~m}$ \\
Coregistration accuracy & $4 \mathrm{bit}(\mathrm{BAQ})$ & $1 / 10 \mathrm{pixel}$ \\
Quantisation & $14 \mathrm{days}$ & $4 \mathrm{bit}(\mathrm{BAQ})$ \\
Repeat cycle & $50 \mathrm{~m} \times 50 \mathrm{~m}$ & $46 \mathrm{days}$ \\
Independent postspacing & & $50 \mathrm{~m} \times 50 \mathrm{~m}$ \\
\hline
\end{tabular}

indicated range of ground-to-volume ratios in case of high temporal decorrelation $\left(\gamma_{\mathrm{tmp}} \approx 0.4\right)$. A separation of the phase centres will only be possible for a narrow range of perpendicular baselines in the order of $800 \mathrm{~m}$. Other baselines and lower temporal coherence will cause significant overlaps of the two probability density functions (pdfs) of the interferometric phase at the extremes of the indicated groundto-volume scattering range. Note that this optimum baseline length will of course depend on the height, the extinction, and the expected range of ground-to-volume ratios of the investigated scenario.

The estimated range of $m$ values in Table 1 assumes a Scots pine forest with a height of $20 \mathrm{~m}$ imaged at an incident angle of $35^{\circ}$. Other scenarios could result in a shift of both the low and high $m$ values. Furthermore, a decrease of the incident angle is expected to increase the ground-tovolume ratios (cf. Section 2.1.1). A positive shift of the $m$ values would significantly improve the inversion performance due to the strong decrease of the interferometric phase centre associated with a slight increase of the ground-to-volume ratio. For medium baselines on the order of $400 \mathrm{~m}$ to $800 \mathrm{~m}$ and for $m$ values in the range of $-5 \mathrm{~dB}$ to $+5 \mathrm{~dB}$, there is an approximately linear phase centre decay of ca. $-0.7 \mathrm{~m} / \mathrm{dB}$ in the investigated example. A possible shift of the $m$-range by $6 \mathrm{~dB}$ to the right would hence increase the measurable height difference from $5 \mathrm{~m}$ to more than $9 \mathrm{~m}$. In this case, the performance estimation predicts a reliable separation of the phase centres even for $\gamma_{\mathrm{tmp}}=0.4$ where the standard deviation of the height errors will be below $\pm 2 \mathrm{~m}( \pm 3.5 \mathrm{~m})$ for a perpendicular baseline of $800 \mathrm{~m}$ (400 m).

High vegetation layers with high total extinction and lower ground-to-volume ratios as well as a reduced temporal coherence may deteriorate the performance. This can be compensated by an increase of the independent postspacing, thereby increasing the number of independent looks available for spatial averaging. The error tubes will become thinner by a factor which is approximately inverse to the square root of the number of looks. The baselines should be again in the order of $800 \mathrm{~m}$ for the investigated Scots pine scenario to enable an optimal separation of the phase centres.

\subsection{ALOS/PaISAR}

This section illustrates the achievable Pol-InSAR performance for ALOS/PalSAR operating in a repeat-pass mode. The relevant system parameters used in the current performance evaluation are summarised in the right column of Table 3. Figure 9 shows the expected height errors for a ground resolution of $50 \mathrm{~m} \times 50 \mathrm{~m}$. As can be seen by comparison with Figure 8 for TerraSAR-L, the height errors will become significantly larger for ALOS/PalSAR. The major reason for the increased phase and height errors is the reduced number of looks due to the small system bandwidth provided by ALOS/PalSAR in the fully polarimetric mode $(14 \mathrm{MHz}$ versus $80 \mathrm{MHz}$ in TerraSAR-L).

It becomes clear that for a ground resolution of $50 \mathrm{~m} \times$ $50 \mathrm{~m}$ the separation of different phase centres with different polarisations will become quite difficult for the indicated range of ground-to-volume ratios. A separation of the phase centres seems to be only possible for baselines on the order of $800 \mathrm{~m}$ if the coherence loss due to temporal decorrelation remains very small $\left(\gamma_{\mathrm{tmp}}>0.8\right.$, red solid tube in Figure 9). A higher coherence loss would already cause a significant overlap of the two probability density functions (pdfs) of the interferometric phase at the extremes of the ground-to-volume range $\left(m_{\min }=-26 \mathrm{~dB}\right.$ and $\left.m_{\max }=-2 \mathrm{~dB}\right)$. As can be seen, this is true for all interferometric baselines. The lowest errors are to be expected for a baseline of ca. $800 \mathrm{~m}$, where we have standard deviations of the height errors of ca. $\pm 2.5 \mathrm{~m}$, $\pm 3.5 \mathrm{~m}$, and $\pm 6 \mathrm{~m}$ for $\gamma_{\mathrm{tmp}}=0.8,0.6$, and 0.4 , respectively, while the separation of the phase centres within the indicated ground-to-volume range is only ca. $5 \mathrm{~m}$. From this, we conclude that a very low accuracy has to be expected for the inversion of the Scots pine forest reference scenario acquired with ALOS/PalSAR. The major reason may be found in the small range bandwidth of $14 \mathrm{MHz}$, which allows only for a small number of looks (between 25.2 looks for $B_{\perp}=200 \mathrm{~m}$ 


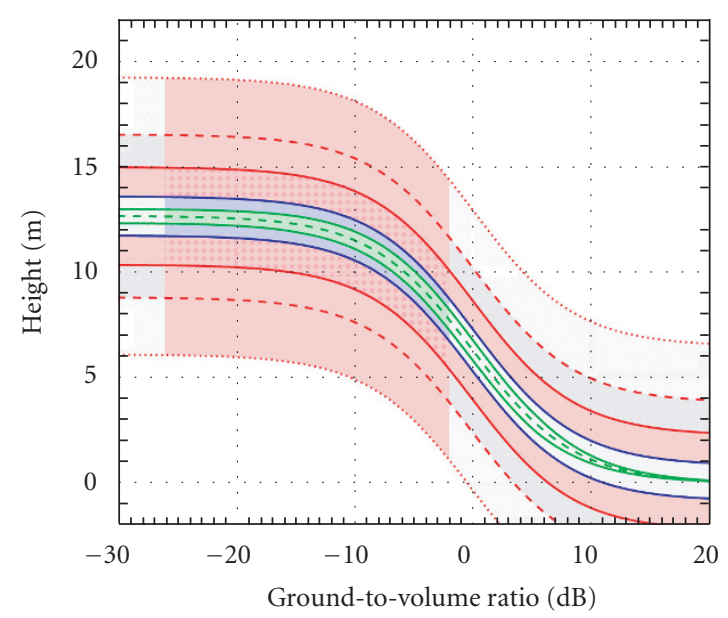

(a)

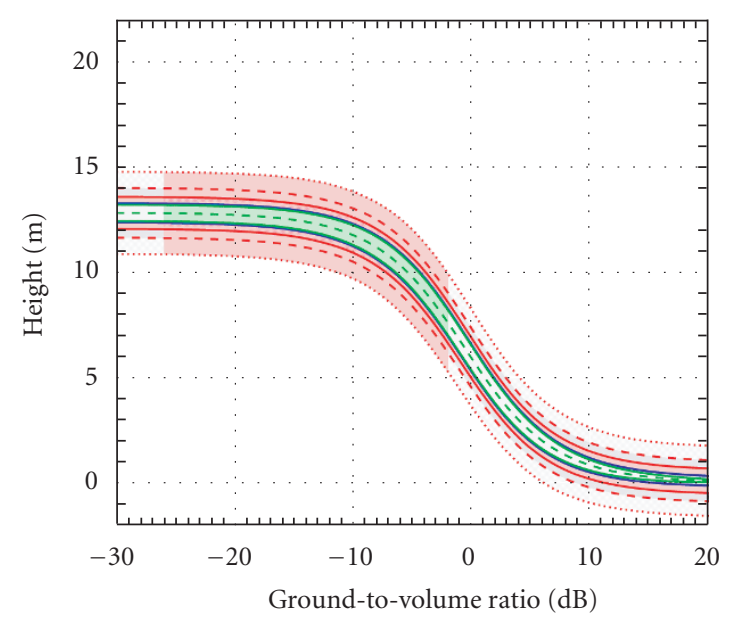

(c)

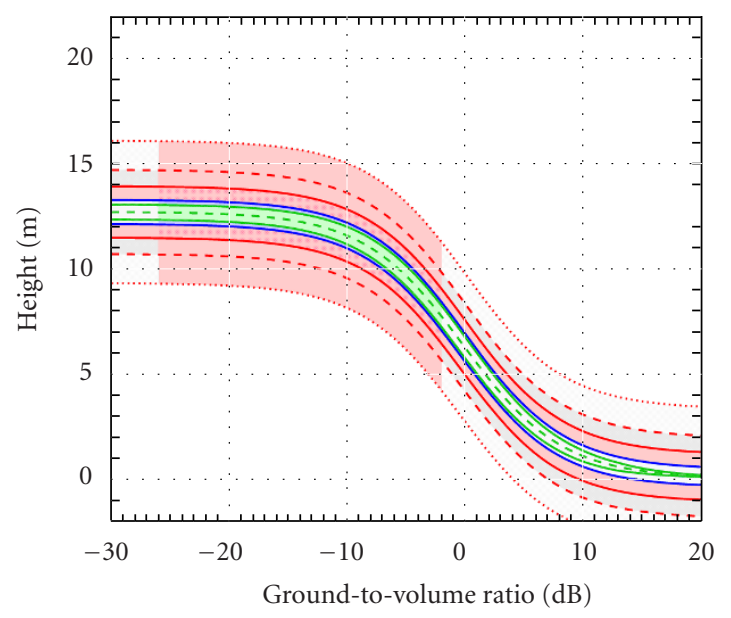

(b)

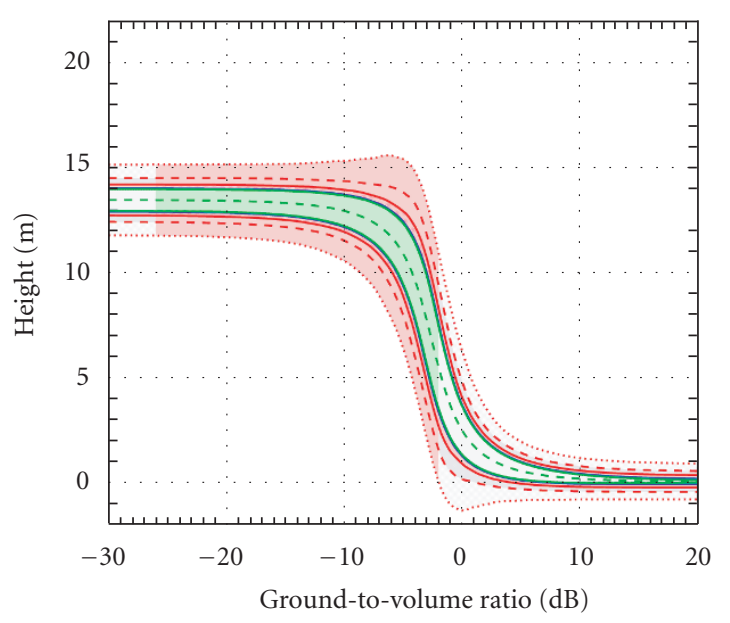

(d)

FIGURE 8: Performance estimation for a TerraSAR-L repeat-pass forest scenario with different interferometric baselines and an independent postspacing of $50 \mathrm{~m} \times 50 \mathrm{~m}$. ((a) $B_{\perp}=200 \mathrm{~m}$; (b) $B_{\perp}=400 \mathrm{~m}$; (c) $B_{\perp}=800 \mathrm{~m}$; (d) $B_{\perp}=1600 \mathrm{~m}$.) The volume height is $20 \mathrm{~m}$, the extinction coefficient is $0.3 \mathrm{~dB} / \mathrm{m}$, and the incident angle is $35^{\circ}$. The indicated baselines are perpendicular to the line of sight. Green tubes show height errors due to volume decorrelation, blue tubes show additional errors due to the limited system accuracy, and red tubes indicate the total errors in case of temporal decorrelation (solid: $\gamma_{\text {temp }}=0.8$, dashed: $\gamma_{\text {temp }}=0.6$, dotted: $\gamma_{\text {temp }}=0.4$ ). The expected range of ground-to-volume ratios ranging from $-26 \mathrm{~dB}$ to $-2 \mathrm{~dB}$ is indicated by the darker areas of the height error tubes.

and 19.5 looks for $B_{\perp}=1600 \mathrm{~m}$ at an independent postspacing of $50 \mathrm{~m} \times 50 \mathrm{~m}$ ). Furthermore, the limited range bandwidth would prohibit the use of larger baselines in case of lower vegetation layers due to a significant increase of baseline decorrelation.

The poor Pol-InSAR performance may be alleviated by an increase of the ground resolution. For example, an increase of the independent postspacing from $50 \mathrm{~m} \times 50 \mathrm{~m}$ to $100 \mathrm{~m} \times 100 \mathrm{~m}$ would decrease the phase errors by a factor of approximately two. In this case, it would become possible to tolerate moderate values of temporal decorrelation $\left(\gamma_{\mathrm{tmp}} \sim 0.6\right)$ in case of appropriately chosen interferometric baselines. In this context, it is also important to note that the predicted improvement with increasing ground resolution depends strongly on the assumption that all errors caused by the time-lag between the acquisitions of the interferometric SAR images can be modelled by a stationary and additive random field with white power spectral density. This implies uncorrelated and homogeneous noise statistics in neighbouring resolution cells. Further studies have to show whether this assumption is justified for all types of temporal scene changes.

\section{SINGLE-PASS MISSION SCENARIOS}

The previous investigations revealed that temporal decorrelation will put a strong limit to the achievable performance in a repeat-pass Pol-InSAR mission scenario. Further 


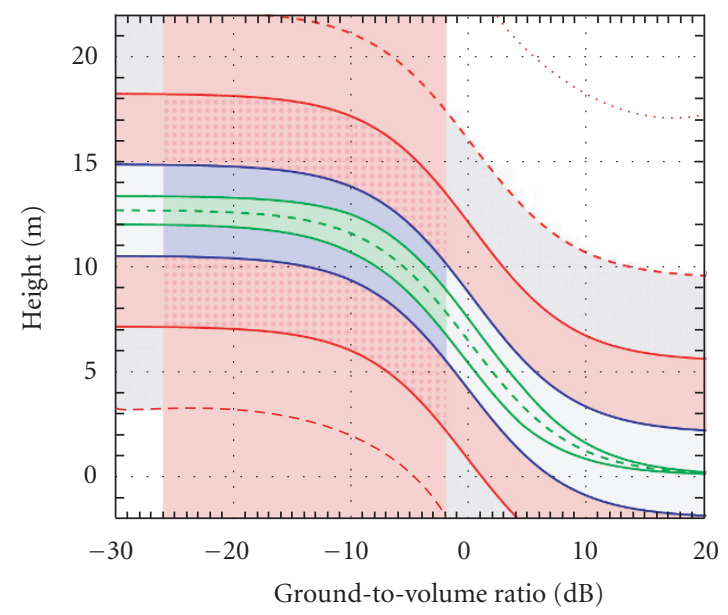

(a)

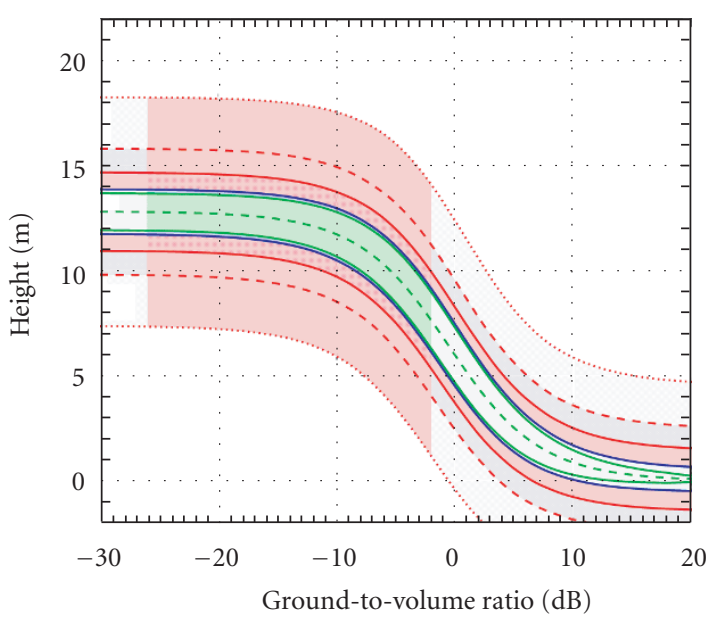

(c)

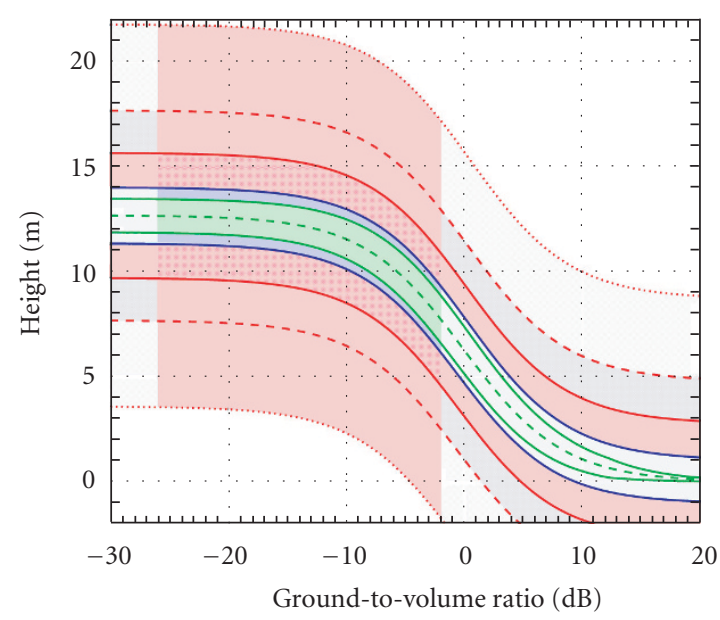

(b)

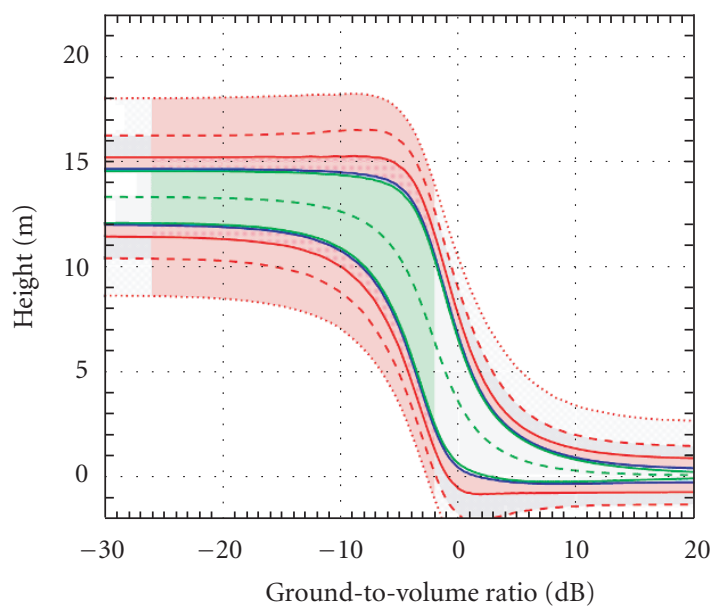

(d)

FIGURe 9: Performance estimation for ALOS/PalSAR repeat-pass scenario with different interferometric baselines and an independent postspacing of $50 \mathrm{~m} \times 50 \mathrm{~m}$. ((a) $B_{\perp}=200 \mathrm{~m}$; (b) $B_{\perp}=400 \mathrm{~m}$; (c) $B_{\perp}=800 \mathrm{~m}$; (d) $B_{\perp}=1600 \mathrm{~m}$.) The volume height is $20 \mathrm{~m}$, the extinction coefficient is $0.3 \mathrm{~dB} / \mathrm{m}$, and the incident angle is $35^{\circ}$. The indicated baselines are perpendicular to the line of sight. Green tubes show height errors due to volume decorrelation, blue areas show additional errors due to the limited SNR, and red areas indicate the errors for temporal decorrelation (solid: $\gamma_{\text {temp }}=0.8$, dashed: $\gamma_{\text {temp }}=0.6$, dotted: $\gamma_{\text {temp }}=0.4$ ). The expected range of ground-to-volume ratios ranging from $-26 \mathrm{~dB}$ to $-2 \mathrm{~dB}$ is indicated by the darker areas of the height error tube.

limitations may arise from distortions of the interferometric phase by atmospheric disturbances [3] and shifts of the polarimetric base by different Faraday rotations during the two satellite passes [46]. To avoid such fundamental limitations, several spaceborne single-pass InSAR mission concepts have been suggested over the last years. While most of these missions have primarily been designed for operation in a singlepolarisation mode, they can be extended/upgraded to provide fully polarimetric capabilities. The first suggestion for such an upgrade was the polarimetric extension of the interferometric cartwheel which has been proposed in 2002 by a joint initiative of CNES and DLR in the framework of the ESA Earth Observation Envelope Programme (VOICE proposal [24]).
One opportunity to acquire interferometric data in a single pass is to use two antennas mounted on a single spacecraft. A prominent example for such a configuration is the Shuttle Radar Topography Mission (SRTM) which was the first and up to now only single-pass cross-track interferometer in space [47]. However, this boom concept suffers from the maximum achievable length of the interferometric baseline, thereby limiting its potential Pol-InSAR application mainly to short wavelength imaging of thick volumes with low extinction [48]. As an alternative, several suggestions have been made to use two or more independent spacecrafts for the simultaneous acquisition of interferometric data with reconfigurable baselines of almost arbitrary length $[22,36,49,50,51,52,53]$. In such a scenario, close 


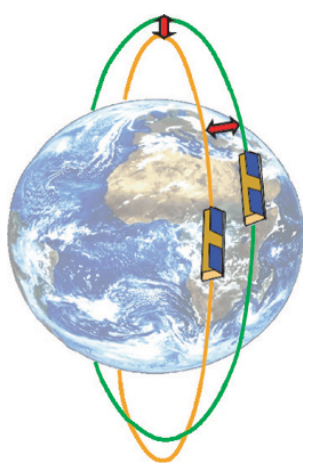

(a)

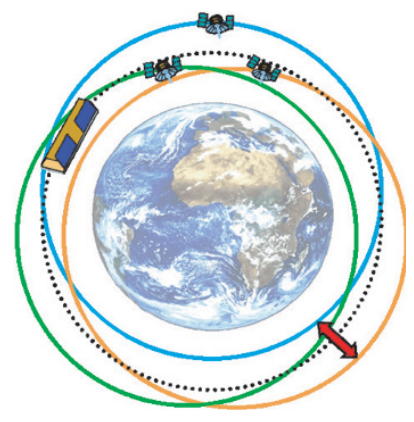

(b)

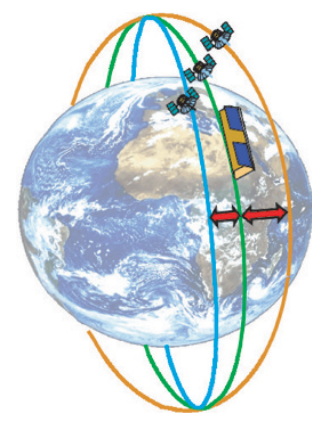

(c)

FIGURE 10: Satellite formations for single-pass cross-track interferometry: (a) fully active tandem in HELIX formation, (b) interferometric cartwheel (semiactive), and (c) semiactive trinodal pendulum (semiactive).

formations will be preferred to avoid baseline decorrelation, temporal decorrelation, and atmospheric disturbances. Multisatellite constellations can be grouped into two major categories: (1) fully active constellations where each satellite has both transmit and receive capabilities and (2) semiactive satellite constellations which combine an active illuminator with several passive receivers.

Fully active SAR constellations use conventional radar satellites flying in close formation to acquire interferometric data during a single pass (cf. Figure 10a). Examples of fully active SAR constellations are twin satellite formations like the Radarsat 2/3 tandem [50] or TanDEM-X [51], as well as multisatellite constellations like the Technology Satellite of the 21st Century (TechSAT, [52]). Fully active constellations have in general a higher sensitivity and flexibility, are less prone to ambiguities, and enable easier phase synchronization like in a ping-pong mode with alternating transmitters or by a direct exchange of radar pulses. Furthermore, they provide also a pursuit monostatic mode as a natural fallback solution in case of problems with orbit control or instrument synchronization.

Semiactive SAR constellations use multiple passive receivers in combination with one active radar illuminator. Passive receivers will enable a cost-efficient implementation of a spaceborne SAR interferometer since the low-power demands and the use of deployable antennas will allow for an accommodation of the payload on low-cost microsatellites. The opportunity to use small and light-weight microsatellites will also significantly reduce the launch costs. The cost advantage is especially pronounced if the receiver constellation is combined with a conventional SAR mission. A first proposal for a semiactive interferometric SAR mission was the interferometric cartwheel [54] and some extensions of this concept have been presented in $[23,36]$. Figure 10 shows two examples of semiactive SAR constellations. The interferometric cartwheel ([22], Figure 10b) and the cross-track pendulum [36] have both been designed to provide one almost constant cross-track baseline across the whole orbit. Alternatives are constellations which provide multiple interferometric baselines at a fixed baseline ratio. Such a multibaseline acquisition will substantially alleviate the problem of resolving phase ambiguities in case of large baselines, but a latitude-based acquisition strategy has to be applied to achieve global coverage [40]. One example for such a configuration is the trinodal pendulum [55], which is shown in Figure 10c. Another example for a multibaseline SAR interferometer is the two-scale cartwheel [56]. Both configurations have been suggested in the framework of a joint DLR/CNES TerraSAR-L cartwheel constellation study, which has been initiated and supported by ESA with the major goal to derive a digital elevation model (DEM) on a global scale [57]. The performance analyses in this study demonstrated that an excellent height accuracy may be achieved by using multibaseline acquisitions in single-polarisation mode [23].

In the following, we will investigate the achievable performance of fully polarimetric SAR constellations. The blue tubes in Figures 8 and 9 may be regarded as first-order approximations of the achievable performance for a potential fully active tandem configuration consisting of either two TerraSAR-L or two ALOS/PalSAR satellites, respectively. Note that the indicated interferometric baselines should be multiplied by a factor of two due to the bistatic operation with only one transmitter. Furthermore, the performance might be slightly worse in case of nonvanishing along-track baselines due to a relative shift of the Doppler spectra.

As an example for a semiactive SAR configuration, we assume an illumination by TerraSAR-L operating in alternating polarisation mode (cf. Table 3 ). In such a scenario, an important issue arises from the small antennas of the passive receivers. The reduced antenna size is a prerequisite for an accommodation of all receiver satellites in one common launcher. The antenna size and its shape are further limited by the maximum momentum that can be handled by a microsatellite. For the scope of the current investigation, we assume a circular aperture with a radius of $1,5 \mathrm{~m}$. As compared to a fully active TerraSAR-L tandem, the reduced size of the receiver apertures will cause a loss of receiver sensitivity 
TABLE 4: Receiver parameters.

\begin{tabular}{lc}
\hline Parameter & Value \\
\hline Antenna size $(\mathrm{Rx})$ & circular: $3 \mathrm{~m}$ \\
Receiver noise figure & $2,5 \mathrm{~dB}$ \\
$\mathrm{Rx}+$ Proc. losses & $2 \mathrm{~dB}$ \\
Losses across swath & $3 \mathrm{~dB}$ (for $40 \mathrm{~km}$ swath) \\
Atmospheric losses & $1 \mathrm{~dB}$ \\
Relative along-track displacement & $1 \mathrm{~km}$ \\
Processed bandwidth & $1200 \mathrm{~Hz}$ \\
Coregistration accuracy & $1 / 8$ pixel \\
\hline
\end{tabular}

by more than $6 \mathrm{~dB}$. Furthermore, the small receiver antennas will also cause a rise of the ambiguity levels, which may limit the unambiguous swath width as well as the operational range to steeper incident angles [40]. Table 4 summarises the parameters for the passive receivers.

A detailed sensitivity analysis has been performed assuming the same scene parameters as for the repeat-pass mission scenarios (cf. Table 1). The predicted variation of the vertical phase centres is shown in Figure 11 together with the associated height errors assuming an independent postspacing of $50 \mathrm{~m} \times 50 \mathrm{~m}$. It is clear that the performance evaluation predicts a very good separation of the phase centres for interferometric baselines in the order of $1 \mathrm{~km}$ to $2 \mathrm{~km}$. For example, a baseline of $1.6 \mathrm{~km}$ and a ground-to-volume scattering range from $-26 \mathrm{~dB}$ to $2 \mathrm{~dB}$ will cause a phase centre separation which exceeds 6 times the standard deviation of the height errors.

The red tubes in Figure 11 show additional errors from a potential temporal decorrelation. Such errors may result from a relative along-track displacement between the passive receivers which will cause a delayed recording of the scattered signals with equal Doppler frequencies. This delay will be approximately 70 milliseconds for a receiver separation of $1 \mathrm{~km}$. Unfortunately, there exist no systematic and quantitative investigations of short-time decorrelation of vegetated areas in L-band. Measurements in X-band indicate decorrelation times below 50 milliseconds for some tree species at moderate windspeeds [58]. Temporal decorrelation is expected to be significantly lower for L-band due to the longer wavelength and the less pronounced scattering from the leaves. For the current performance analysis, we made hence the reasonable assumption that the temporal coherence exceeds $\gamma_{\text {tmp }}>0.9$ for time-lags below 100 milliseconds. Note that it is also possible to completely avoid such a residual temporal decorrelation by minimizing the along-track displacement between the satellites. For example, a slight change of the eccentricity vectors in the trinodal pendulum will cause a small vertical displacement at the northern and southern turns (Helix configuration, see also [59]). Such a configuration separates the receiver satellites by a combination of vertical and horizontal cross-track displacements, and there will be no crossing of the satellite orbits. The satellites may hence be shifted arbitrarily along their orbits without any collision risk. This concept is therefore well suited to provide zero along-track baselines for the desired scene positions. A detailed orbit analysis of this satellite formation can be found in [60].

As mentioned before, the Pol-InSAR performance will strongly depend on the scatterer characteristics, like volume height, extinction, and scattering coefficient. Each of these parameters will influence the selection of an appropriate baseline length. Hence, any optimisation of the satellite constellation will require some a priori information about the scenes to be imaged. Especially the expected range of volume heights has to be known a priori in order to determine optimised baseline lengths. As an alternative, the operational range of the SAR interferometer can be significantly extended by using interferometric data acquisition with more than one baseline. One example for such a configuration is the tri-nodal pendulum which is shown in Figure 10c. This constellation offers the unique feature to acquire three interferograms with three different baselines in a single pass. Such a multibaseline acquisition may significantly alleviate the process of model inversion. Note that the three interferograms obtained with the trinodal pendulum will not be completely independent, since the phase of the third interferogram is determined by the phase of the two other interferograms. Nevertheless, the simultaneous mapping with three different baselines will allow for the selection of an optimum baseline for different volume heights without the necessity to rearrange the satellite constellation. A combined evaluation of the interferometric data from all interferograms may further enhance the system performance and parameter inversion accuracy. Indeed, it has been shown that the availability of more than one baseline increases the performance of vegetation parameter estimates in terms of Pol-InSAR [31, 61]. Further, it allows the inversion of more complex models-such as, for example, three-layer models-providing more information about the vertical structure of vegetation $[31,61]$.

\section{DISCUSSION}

We have shown that the performance of Pol-InSAR systems depends on the measurable phase centre separation caused by a mixture of combined surface and volume scattering. The optimum configuration would then provide maximum phase centre separation combined with high coherence. In this way, important secondary products such as mean forest height may be estimated using a single-baseline, singlewavelength sensor with accuracies approaching 10\%. However, this performance is degraded by system and temporal decorrelation effects. We have quantified the combined effects of system and scattering coherence losses by introducing a new phase tube representation. This concept can then be used to analyse the performance of practical Pol-InSAR systems.

Our performance analysis in Section 3 revealed that the accuracy of Pol-InSAR products acquired in a repeatpass mission scenario will be significantly affected by temporal decorrelation. Assuming that errors from temporal 


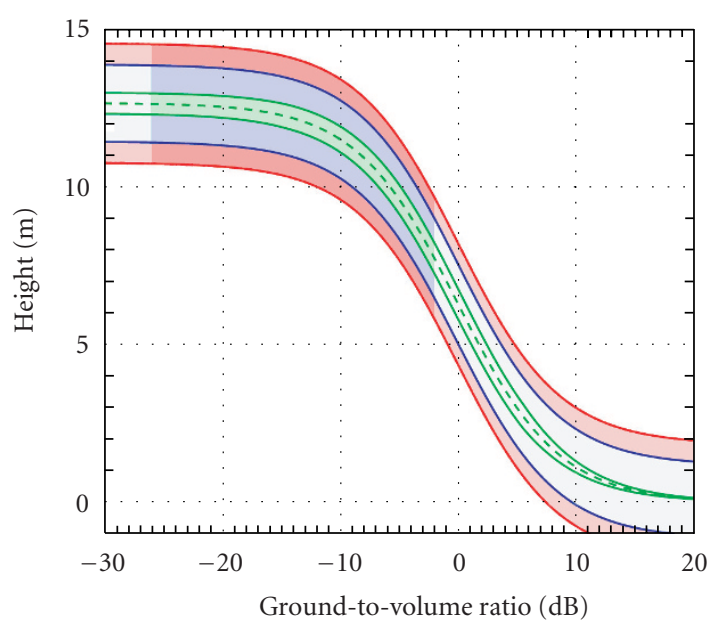

(a)

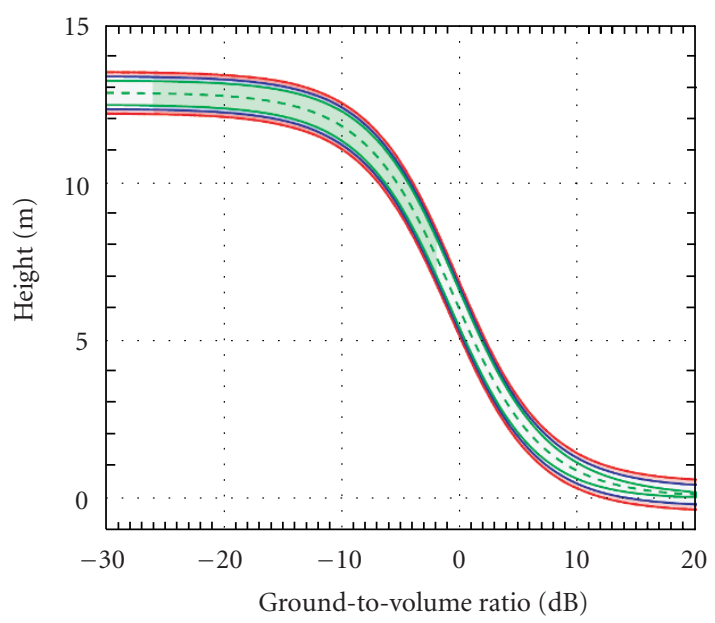

(c)

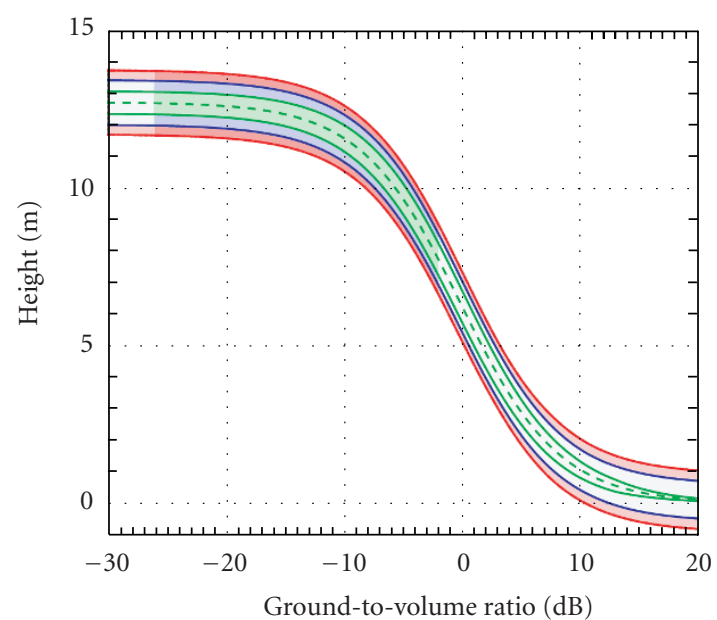

(b)

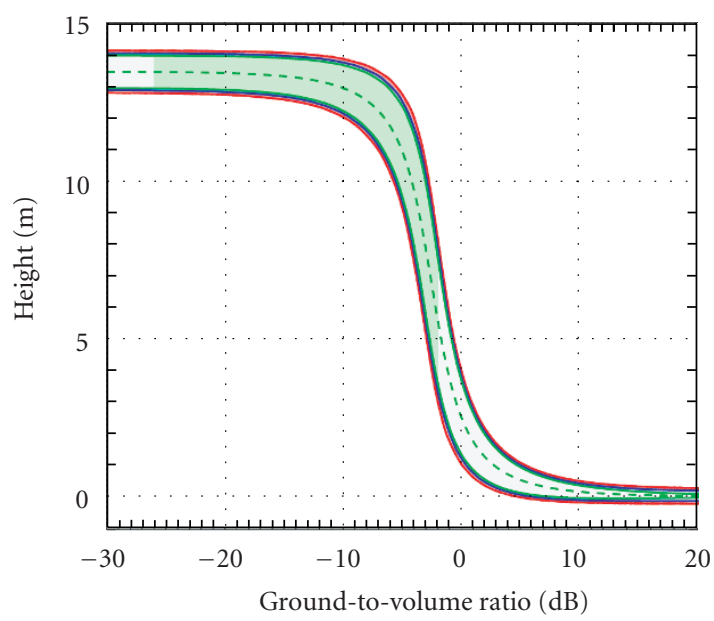

(d)

FIGURE 11: Performance estimation for fully polarimetric TerraSAR-L cartwheel constellation with different interferometric baselines. ((a) $B_{\perp}=400 \mathrm{~m}$; (b) $B_{\perp}=800 \mathrm{~m}$; (c) $B_{\perp}=1600 \mathrm{~m}$; (d) $B_{\perp}=3200 \mathrm{~m}$.) The volume height is $20 \mathrm{~m}$ and the extinction coefficient is $0.3 \mathrm{~dB} / \mathrm{m}$. The independent postspacing is $50 \mathrm{~m} \times 50 \mathrm{~m}$ and the given baselines are perpendicular to the line of sight. Green tubes show height errors due to volume decorrelation, blue areas show additional errors due to the limited system accuracy, and red areas indicate the errors for temporal decorrelation $\left(\gamma_{\text {temp }}=0.9\right)$. The expected range of ground-to-volume ratios ranging from $-26 \mathrm{~dB}$ to $-2 \mathrm{~dB}$ is indicated by the darker areas of the height error tube.

decorrelation can be treated as additive stationary white noise, it is possible to reduce the errors in the final interferogram by spatial averaging. Further studies have to show whether this assumption is justified for the different temporal error sources like wind (systematic versus random shift of twigs and leaves), defoliation, moisture changes, and so forth. As discussed in Section 2, it is advisable to select system bandwidths which are as high as possible since this will increase the number of independent looks for a given independent postspacing of the final product. In this way, it is also possible to reduce the contributions from volume decorrelation in case of large baselines. The major results of the performance evaluation for each of the analysed satellite missions may be summarised as follows.

\section{TerraSAR-L}

A reliable separation of the phase centres will become possible for the Scots pine forest reference scenario at low-tomoderate temporal decorrelation. Higher temporal decorrelation may require an increase of the spatial resolution in the final Pol-InSAR product. The performance will further strongly depend on the range of ground-to-volume ratios provided by the different polarisations. This range is expected to improve for lower incident angles.

\section{ALOS/PaISAR}

The performance model predicts a rather poor inversion accuracy for the Scots pine reference scenario due to the low 
number of independent looks resulting from the small system bandwidth of PalSAR (only $14 \mathrm{MHz}$ for fully polarimetric operation). An acceptable performance may be achieved by increasing the independent postspacing to $100 \mathrm{~m} \times 100 \mathrm{~m}$. In this case, a separation of the phase centres would become possible in case of low-to-moderate temporal decorrelation, but it has to be noted that the coherence values may often drop below these values due to the long repeat cycle of ALOS (46 days). Potential orbit manoeuvres to change the orbit for reduced repeat cycles for a limited mission period have been investigated in [48].

Further critical issues for a repeat-pass mission scenario arise from atmospheric disturbances and a relative shift of the polarisation bases between the acquisitions of the interferometric images by different amounts of Faraday rotation. Atmospheric disturbances may cause a space variant phase offset between the two interferometric channels. This interferometric phase disturbance will have a high degree of spatial correlation [3]. It is hence expected that such an offset will mainly affect estimates of the ground topography phase while leaving estimates of the residual model parameters untouched.

Faraday rotation may become several tens of degrees at Lband for solar maximum [46]. For single-pass InSAR configurations, it affects both interferometric channels in the same way, so that only absolute effects become an issue. Because Pol-InSAR applications like forest height estimation require an absolute knowledge of the actual polarisation state only for qualitative interpretation, the accuracy of conventional quad-pol Faraday rotation calibration algorithms is sufficient [62]. For repeat-pass InSAR configurations, the calibration requirements become more critical as different Faraday rotation angles in the two observations may introduce a coherence degradation biasing any height estimation. In this case, the tolerance level of residual polarimetric phase errors for reliable model inversion has still to be analysed in detail.

Regarding now conventional polarimetric calibration, the Pol-InSAR requirements are-relatively-relaxed. High cross-talk values compress the apparent ground-to-volume ratio $m$ value range directly affecting inversion performance. However, the dynamic range of $m$ according to the simulations used in this study is less than $20 \mathrm{~dB}$ so that as long as the residual cross-talk values are better than this, the effect of cross-talk on forest height inversion can be ignored [63]. Relative polarimetric phase calibration is not critical for PolInSAR applications as long as they do not introduce phase offsets between the individual baselines.

Finally, for the realisation of small baselines (in order to control volume decorrelation on a mission basis), the orbit control should be accurate enough to allow orbit maintenance within $10 \%-20 \%$ of the shortest baselines. This means that for 500-1000-meter baselines (i.e., about 10\% of the critical baseline for the ALOS/PalSAR configuration), orbital tubes (i.e., orbit maintenance accuracy) of about 50-100 meter have to be established. A further issue are baseline estimation errors which will introduce two kinds of height errors: a low-frequency topography tilt and a miss-scaling of the relative terrain variation due to the erroneous phase-to-height conversion. They are critical for conventional InSAR DEM generation, and affect most Pol-InSAR applications in the same way. Hence, the Pol-InSAR requirements on baseline/orbit estimation are fulfilled by the conventional InSAR requirements. A calibration of the Pol-InSAR products derived from the model inversion process will also be complicated by the unknown levels of temporal decorrelation which could be mistaken as volume decorrelation in case of large interferometric baselines.

A promising alternative to the conventional repeat-pass mission scenarios with a single satellite are fully polarimetric satellite formations which enable a quasisimultaneous acquisition of the interferometric channels with a flexible imaging geometry. Such bi- and multistatic satellite constellations have been introduced and analysed in Section 4. Due to the low level of temporal decorrelation, a good performance is predicted providing a sufficient vertical phase centre separation even in case of a narrow range of ground-tovolume scattering ratios $m$. If required, residual errors due to short-term temporal decorrelation can be avoided by an appropriate orbit/formation design. Furthermore, the influence of baseline errors is reduced by a factor of two in a bistatic single-pass mission scenario. The simultaneous data acquisition will also enable a direct evaluation of the GPS phase carrier signals to determine the interferometric baselines (i.e., relative satellite position) with an accuracy below $1 \mathrm{~cm}$ [51]. An important issue may arise from oscillator phase noise which will introduce a low-frequency azimuth modulation of the interferometric phase. This error will therefore mainly influence estimates of the ground topography phase $\varphi_{0}$ while leaving other Pol-InSAR parameters like volume height $h_{v}$, ground-to-volume scattering ratio $m$, and extinction $\sigma$ mainly untouched. Oscillator phase errors may, for example, be avoided by an appropriate synchronisation link.

The baseline length for optimum Pol-InSAR performance will strongly depend on the volume height to be imaged. Hence, the selection of an optimised Pol-InSAR satellite constellation will require some a priori knowledge of the volume height. An alternative is the interferometric data acquisition with multiple baselines. Semiactive satellite constellations like the trinodal pendulum enable the acquisition of multiple interferograms with fixed baseline ratios in a single pass. Small cross-track baselines are well suited to avoid phase wrapping problems for high vegetation layers while large baselines will allow for an improved accuracy in areas with low vegetation. It is expected that an excellent performance can be achieved by combining the interferometric data from several baselines.

A further opportunity in using a single-pass interferometer is a staggered acquisition of different polarisations. Such a "repeat-pass polarimeter" will acquire the full scattering matrices in several satellite passes. Such a scheme could be of special interest for a semiactive satellite constellation like the interferometric cartwheel or the trinodal pendulum, since it will allow for the acquisition of a fully polarimetric and interferometric data set without the necessity to increase the complexity of the receiver hardware by a second polarimetric 
channel. A further advantage is the reduced susceptibility to ambiguities, since each acquisition will only use a single polarisation in the transmitter. In case of an appropriately designed microsatellite with circular antennas, the different polarisations may be easily acquired one after the other by a rotation of the microsatellites and an appropriate switching of the transmitted polarisation plane. This will require one (in case of using alternating Tx polarisations) or two (in case of single Tx polarisations) additional passes. The latter case will allow for an improvement of the SNR by $3 \mathrm{~dB}$ due to the higher duty cycle. The impact of the coherence loss between the polarimetric channels due to temporal decorrelation has still to be investigated, but it may be speculated that the PolInSAR performance will be less affected than by a temporal decorrelation between the interferometric channels.

\section{ACKNOWLEDGMENTS}

This study has been supported by the European Space Agency (Contract no. 17893/03/I-LG). We thank the anonymous reviewers for their constructive comments and greatly appreciate their valuable suggestions to improve the quality of the paper.

\section{REFERENCES}

[1] R. Bamler and P. Hartl, "Synthetic aperture radar interferometry," Inverse Problems, vol. 14, no. 4, pp. R1-R54, 1998.

[2] P. A. Rosen, S. Hensley, I. R. Joughin, et al., "Synthetic aperture radar interferometry," Proc. IEEE, vol. 88, no. 3, pp. 333$382,2000$.

[3] R. F. Hanssen, Radar Interferometry: Data Interpretation and Error Analysis, Kluwer Academic, Dordrecht, The Netherlands, 2001.

[4] J. O. Hagberg, L. M. H. Ulander, and J. Askne, "Repeat-pass SAR interferometry over forested terrain," IEEE Trans. Geosci. Remote Sensing, vol. 33, no. 2, pp. 331-340, 1995.

[5] J. I. H. Askne, P. B. G. Dammert, L. M. H. Ulander, and G. Smith, "C-band repeat-pass interferometric SAR observations of the forest," IEEE Trans. Geosci. Remote Sensing, vol. 35, no. 1, pp. 25-35, 1997.

[6] R. N. Treuhaft, S. N. Madsen, M. Moghaddam, and J. J. van Zyl, "Vegetation characteristics and underlying topography from interferometric radar," Radio Science, vol. 31, no. 6, pp. 1449-1485, 1996.

[7] R. N. Treuhaft and P. R. Siqueira, "The vertical structure of vegetated land surfaces from interferometric and polarimetric radar," Radio Science, vol. 35, no. 1, pp. 141-177, 2000.

[8] W.-M. Boerner, H. Mott, E. Lüneburg, et al., "Polarimetry in radar remote sensing: basic and applied concepts," in Principles and Applications of Imaging Radar, F. M. Henderson and A. J. Lewis, Eds., vol. 2 of Manual of Remote Sensing, chapter 5, pp. 271-358, John Willey \& Sons, New York, NY, USA, 3rd edition, 1998.

[9] S. R. Cloude, K. P. Papathanassiou, and E. Pottier, "Radar polarimetry and polarimetric interferometry," IEICE Transactions on Electronics, vol. E84-C, no. 12, pp. 1814-1822, 2001.

[10] S. R. Cloude and K. P. Papathanassiou, "Polarimetric SAR interferometry," IEEE Trans. Geosci. Remote Sensing, vol. 36, no. 5, pp. 1551-1565, 1998.

[11] S. R. Cloude and K. P. Papathanassiou, "Polarimetric optimisation in radar interferometry," Electronics Letters, vol. 33, no. 13, pp. 1176-1178, 1997.
[12] K. P. Papathanassiou and S. R. Cloude, "Single-baseline polarimetric SAR interferometry," IEEE Trans. Geosci. Remote Sensing, vol. 39, no. 11, pp. 2352-2363, 2001.

[13] S. R. Cloude and K. P. Papathanassiou, "Three-stage inversion process for polarimetric SAR interferometry," IEE Proceedings - Radar, Sonar and Navigation, vol. 150, no. 3, pp. 125-134, 2003.

[14] K. P. Papathanassiou, I. Hajnsek, T. Mette, and A. Moreira, "Model based forest parameter estimation from single baseline Pol-in-SAR data: the Fichtelgebirge test case," in Proc. Workshop on Applications of SAR Polarimetry and Polarimetric Interferometry (POLinSAR '03), ESA-ESRIN, Frascati, Italy, January 2003, http://earth.esa.int/polinsar.

[15] I. Woodhouse, S. R. Cloude, C. Hutchinson, and K. P. Papathanassiou, "Evaluating PolInSAR tree height and topography retrievals in glen affric," in Proc. Workshop on Applications of SAR Polarimetry and Polarimetric Interferometry (POLinSAR '03), ESA-ESRIN, Frascati, Italy, January 2003, http://earth.esa.int/polinsar.

[16] A. D. Friend, "The prediction and physiological significance of tree height," in Vegetation Dynamics and Global Change, A. M. Solomon and H. H. Shugart, Eds., pp. 101-115, Chapman \& Hall, New York, NY, USA, 1993.

[17] C. D. Oliver and B. C. Larson, Forest Stand Dynamics, McGraw-Hill, New York, NY, USA, 1990.

[18] T. E. Avery and H. E. Burkhart, Forest Measurements, McGraw-Hill, New York, NY, USA, 1994.

[19] M. Nilsson, "Estimation of tree heights and stand volume using an airborne lidar system," Remote Sensing of Environment, vol. 56, no. 1, pp. 1-7, 1996.

[20] T. Mette, K. P. Papathanassiou, I. Hajnsek, and R. Zimmermann, "Forest biomass estimation using polarimetric SAR interferometry," in Proc. IEEE International Geoscience and Remote Sensing Symposium (IGARSS '02), vol. 2, pp. 817-819, Toronto, Canada, June 2002.

[21] T. Mette, I. Hajnsek, K. P. Papathanassiou, and R. Zimmermann, "Above ground forest biomass estimation using fully polarimetric / interferometric radar data," in Proc. Workshop on Applications of SAR Polarimetry and Polarimetric Interferometry (POLinSAR '03), ESA-ESRIN, Frascati, Italy, January 2003, http://earth.esa.int/polinsar.

[22] D. Massonnet, "Capabilities and limitations of the interferometric cartwheel," IEEE Trans. Geosci. Remote Sensing, vol. 39, no. 3, pp. 506-520, 2001.

[23] M. Zink, G. Krieger, and T. Amiot, "Interferometric performance of a cartwheel constellation for TerraSAR-L," in Proc. FRINGE Workshop 2003, Frascati, Italy, December 2003.

[24] D. Massonnet, A. Moreira, R. Bamler, and J. C. Souyris, Voice: Volumetric Interferometry Cartwheel Experiment, Proposal to the European Space Agency for a mission to retrieve globally vegetation structure parameters and above ground forest biomass using a passive polarimetric SAR interferometry Cartwheel configuration, January 2002.

[25] R. Touzi, A. Lopes, J. Bruniquel, and P. W. Vachon, "Coherence estimation for SAR imagery," IEEE Trans. Geosci. Remote Sensing, vol. 37, no. 1, pp. 135-149, 1999.

[26] J.-S. Lee, K. P. Papathanassiou, T. L. Ainsworth, M. R. Grunes, and A. Reigber, "A new technique for noise filtering of SAR interferometric phase images," IEEE Trans. Geosci. Remote Sensing, vol. 36, no. 5, pp. 1456-1465, 1998.

[27] J.-S. Lee, K. W. Hoppel, S. A. Mango, and A. R. Miller, "Intensity and phase statistics of multilook polarimetric and interferometric SAR imagery," IEEE Trans. Geosci. Remote Sensing, vol. 32, no. 5, pp. 1017-1028, 1994. 
[28] F. T. Ulaby and M. C. Dobson, Handbook of Radar Scattering Statistics for Terrain, Artech House, Norwood, Mass, USA, 1989.

[29] S. R. Cloude, D. G. Corr, and M. L. Williams, "Target detection beneath foliage using polarimetric Synthetic Aperture Radar interferometry," Waves in Random Media, vol. 14, no. 2, pp. S393-S414, 2004.

[30] K. P. Papathanassiou and S. R. Cloude, "The effect of temporal decorrelation on the inversion of forest parameters from Pol-InSAR data," in Proc. IEEE International Geoscience and Remote Sensing Symposium (IGARSS '03), vol. 3, pp. 14291431, Tolouse, France, July 2003.

[31] S. R. Cloude and M. L. Williams, "A coherent EM scattering model for dual baseline POLInSAR," in Proc. IEEE International Geoscience and Remote Sensing Symposium (IGARSS '03), vol. 3, pp. 1423-1425, Tolouse, France, July 2003.

[32] "Pol-InSAR Product and Technology Assessment," Tech. Rep., Polarimetric and Interferometric Mission and Application Study, ESA Contract No 17893/03/ILG, May 2004.

[33] H. A. Zebker and J. Villasenor, "Decorrelation in interferometric radar echoes," IEEE Trans. Geosci. Remote Sensing, vol. 30, no. 5, pp. 950-959, 1992.

[34] D. Just and R. Bamler, "Phase statistics of interferograms with applications to synthetic aperature radar," Applied Optics, vol. 33, no. 20, pp. 4361-4368, 1994.

[35] J. C. Curlander and R. N. McDonough, Synthetic Aperture Radar: Systems and Signal Processing, Jon Wiley \& Sons, New York, NY, USA, 1991.

[36] G. Krieger, H. Fiedler, J. Mittermayer, K. P. Papathanassiou, and A. Moreira, "Analysis of multistatic configurations for spaceborne SAR interferometry," IEE Proceedings - Radar, Sonar and Navigation, vol. 150, no. 3, pp. 87-96, 2003.

[37] I. H. McLeod, I. G. Cumming, and M. S. Seymour, "ENVISAT ASAR data reduction: impact on SAR interferometry," IEEE Trans. Geosci. Remote Sensing, vol. 36, no. 2, pp. 589-602, 1998.

[38] J. Max, "Quantizing for minimum distortion," IEEE Trans. Inform. Theory, vol. 6, no. 1, pp. 7-12, 1960.

[39] C. Parraga-Niebla and G. Krieger, "Optimization of blockadaptive quantization for SAR raw data," Space Technology, vol. 23, no. 2-3, pp. 131-141, 2003.

[40] G. Krieger, "Performance Analysis for TerraSAR-L Cartwheel Constellation," TN to ESA TS-SW-ESA-SY-0002, ESTEC, Noordwijk, November 2003.

[41] M. Zink, "The TERRASAR-L interferometric mission objectives," in Proc. FRINGE Workshop 2003, ESA-ESRIN, Frascati, Italy, December 2003.

[42] M. Abramowitz and I. Stegun, Handbook of Mathematical Functions, Dover, New York, NY, USA, 1965.

[43] E. Rodriguez and J. M. Martin, "Theory and design of interferometric synthetic aperture radars," IEE Proceedings F, Radar and Signal Processing, vol. 139, no. 2, pp. 147-159, 1992.

[44] H. Kimura and N. Itoh, "ALOS PALSAR: The Japanese second-generation spaceborne SAR and its application," in Proc. Society of Photo-Optical Instrumentation Engineers, vol. 4152, pp. 110-119, 2002.

[45] ESA TerraSAR System Requirements Document, TS-RS-ESASY-0002, 19/01/2004.

[46] P. A. Wright, S. Quegan, N. S. Wheadon, and C. D. Hall, "Faraday rotation effects on L-band spaceborne SAR data," IEEE Trans. Geosci. Remote Sensing, vol. 41, no. 12, pp. 27352744, 2003

[47] M. Werner, "Shuttle radar topography mission (SRTM), mission overview," Journal of Telecommunication (Frequenz), vol. 55, no. 3-4, pp. 75-79, 2001.
[48] Technical Note to Polarimetric and Interferometric Mission and Application Study, ESA Contract No 17893/03/I-LG, 2004.

[49] H. A. Zebker, T. G. Farr, R. P. Salazar, and T. H. Dixon, "Mapping the world's topography using radar interferometry: the TOPSAT mission," Proc. IEEE, vol. 82, no. 12, pp. 1774-1786, 1994.

[50] R. Girard, P. F. Lee, and K. James, "The RADARSAT-2\&3 topographic mission: an overview," in Proc. IEEE International Geoscience and Remote Sensing Symposium (IGARSS '02), vol. 3, pp. 1477-1479, Toronto, Canada, June 2002.

[51] A. Moreira, G. Krieger, I. Hajnsek, et al., "TanDEM-X: a TerraSAR-X add-on satellite for single-pass SAR interferometry," in Proc. IEEE International Geoscience and Remote Sensing Symposium (IGARSS '04), vol. 2, pp. 1000-1003, Anchorage, Alaska, USA, September 2004, (see also DLR Doc. No. 20033472739, prepared by DLR-HR, EADS Astrium GmbH and Infoterra $\mathrm{GmbH}$ in response to the DLR Call for Proposals for a National Earth Observation Mission, November 2003).

[52] M. Martin, P. Klupar, S. Kilberg, and J. Winter, TechSat 21 and Revolutionizing Space Missions Using Microsatellites, American Institute of Aeronautics and Astronautics, Prescott, Ariz, USA, 2001.

[53] G. Séguin and R. Girard, "Interferometric missions using small SAT SAR satellites," in Proc. ISPRS Comission I MidTerm Symposium in Conjunction with Pecora 15/Land Satellite Information IV Conference, Denver, Colo, USA, November 2002.

[54] D. Massonnet, "Roue Interférométrique," French Patent 236910D17306RS, April 1998.

[55] H. Fiedler and G. Krieger, "Formation flying concepts," in Proc. 1st Progress Meeting of ESA TerraSAR-L Cartwheel Constellation Study, Toulouse, France, July 2003.

[56] J. Fourcade, "Flight dynamic mission analysis," in Proc. Final Meeting of ESA TerraSAR-L Cartwheel Constellation Study, November 2003.

[57] M. Zink, "Definition of the TerraSAR-L Cartwheel Constellation," ESA TS-SW-ESA-SY-0002.

[58] R. M. Narayanan, D. W. Doerr, and D. C. Rundquist, "Temporal decorrelation of X-band backscatter from wind-influenced vegetation," IEEE Trans. Aerosp. Electron. Syst., vol. 28, no. 2, pp. 404-412, 1992.

[59] A. Moreira, G. Krieger, and J. Mittermayer, "Comparison of several bistatic SAR configurations for spaceborne SAR interferometry," in Proc. IEEE International Geoscience and Remote Sensing Symposium (IGARSS '01), Sydney, Australia, July 2001, (see also Proc. ASAR Workshop, Saint-Hubert, Quebec 2001 and US-Patent No. 6,677,884 B2).

[60] H. Fiedler, G. Krieger, and F. Jochim, "Close formation flight of micro-satellites for SAR interferometry," in Proc. 2nd International Symposium on Formation Flying Missions \& Technologies, Washington, DC, USA, September 2004, (see also Proc. 18th International Symposium on Space Flight Dynamics, Munich, 2004).

[61] S. R. Cloude, "Robust parameter estimation using dual baseline polarimetric SAR interferometry," in Proc. IEEE International Geoscience and Remote Sensing Symposium (IGARSS '02), vol. 2, pp. 838-840, Toronto, Canada, June 2002.

[62] A. Freeman, "Calibration of linearly polarized polarimetric SAR data subject to Faraday rotation," IEEE Trans. Geosci. Remote Sensing, vol. 42, no. 8, pp. 1617-1624, 2004.

[63] S. R. Cloude, "Calibration requirements for forest parameter estimation using POLinSAR," in Proc. CEOS Working Group on Calibration/Validation SAR Workshop, London, UK, September 2002. 
Gerhard Krieger received the Dipl.-Ing. (M.S.) and the Dr.-Ing. (Ph.D.) degrees in electrical and communication engineering from the Technical University of Munich, Germany, in 1992 and 1999, respectively. From 1992 to 1999, he was a Scientist at the Ludwig-Maximilians University, Munich, where he worked in several interdisciplinary research projects related to the modelling of biological and technical vi-

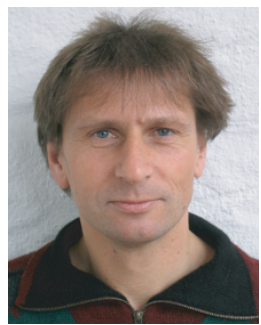
sion systems by applying higher-order statistics and nonlinear systems theory. In 1999, he joined the Microwave and Radar Institute (HR) of the German Aerospace Center (DLR), Oberpfaffenhofen, Germany. From 1999 to 2001, he developed signal processing algorithms for an innovative forward-looking radar (SIREV). Since 2001, he has been the Head of the New SAR Missions Group at DLR (HR). His technical interests include the development and analysis of innovative SAR system concepts based on bi- and multistatic satellite formations, digital beamforming, SAR interferometry, and tomography, as well as advanced signal and image processing techniques.

\section{Konstantinos Panagiotis Papathanassiou} received the Dipl.-Ing. degree (honors) in 1994 and the Dr. degree (honors) in 1999 from the Technical University of Graz, Austria. From 1992 to 1994 , he was with the Institute for Digital Image Processing (DIBAG) of Joanneum Research, Graz, Austria. Between 1995 and 1999, he worked at the Microwaves and Radar Institute (HR) of the German Aerospace Center (DLR),

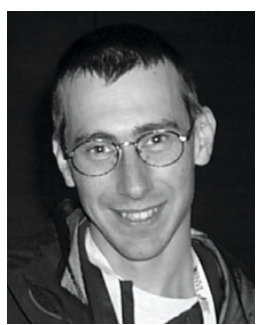
Oberpfaffenhofen, Germany. From 1999 to 2000, he was an EU Postdoctoral Fellow with Applied Electromagnetics (AEL) in Saint Andrews, Scotland. Since October 2000, he has been again with the Microwaves and Radar Institute (HR) of the German Aerospace Center (DLR). Actually he is within the Pol-InSAR Group working on polarimetric SAR interferometry and the development of inversion algorithms for physical parameters from SAR data. His main research interests are in polarimetric and interferometric processing and calibration techniques, polarimetric SAR interferometry, and the quantitative parameter estimation from SAR data. He has more than 50 publications in international journals, conferences, and workshops. He was awarded the IEEE GRSS IGARSS Symposium Prize Paper Award in 1998, the Best Paper Award of the European SAR Conference in 2002, and the DLR Science Award in 2002.

Shane R. Cloude was born in Chatham, England, in 1959. He obtained his B.S. degree (honors) from the University of Dundee, Scotland (1981), and his Ph.D. degree from the University of Birmingham, England (1987), both in electrical and electronic engineering. He worked at the Royal Signals and Radar Establishment (RSRE) in Malvern, England, before taking up teaching and research positions at the Universi-

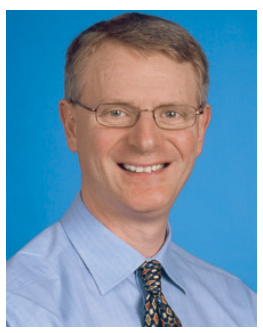
ties of Dundee in Scotland, York in England, and Nantes in France. In 1996, he was the recipient of a fellowship from the Alexander von Humboldt Foundation in Germany, working in collaboration with DLR on the development of Polarimetric SAR Interferometry. Between 1997 and 2004, he was the Director of Applied
Electromagnetics (AELc), a research consultancy company based in Scotland, undertaking contract research in radar and antenna design for industry, government, and defence laboratories. In 2004, he was appointed to the DSTO Chair in Microwave Radar at the University of Adelaide in Australia. Shane Cloude is a Fellow of the IEEE (2001) and an Associate Editor of the IEEE Transactions on Geoscience and Remote Sensing. 